\title{
Anatomy of a high-mass star forming cloud: The G24.78+0.08 (proto)stellar cluster
}

\author{
R. Cesaroni ${ }^{1}$, C. Codella ${ }^{2}$, R. S. Furuya ${ }^{1}$, and L. Testi ${ }^{1}$ \\ 1 Osservatorio Astrofisico di Arcetri, INAF, Largo E. Fermi 5, 50125 Firenze, Italy \\ 2 Istituto di Radioastronomia, CNR, Sezione di Firenze, Largo E. Fermi 5, 50125 Firenze, Italy
}

Received 31 October 2002 / Accepted 21 January 2003

\begin{abstract}
We present the results of an interferometric and single-dish study of G24.78+0.08, a region associated with highmass star formation. Observations have been carried out in several molecular species, which are suitable to trace environments with different densities and temperatures. Evidence for this region to contain a cluster of very young massive stellar objects has been presented in a previous paper (Furuya et al. 2002). We suggest that the embedded stars might be too young to have affected the surrounding molecular cloud significantly on a large scale. This gives us the opportunity to investigate the configuration of the cloud as it was prior to the star formation episode. We assess that the (proto)stellar cluster lies at the center of a molecular clump with diameter of $\sim 2 \mathrm{pc}$ : to a good approximation this may be described as a spherically symmetric clump with density profile of the type $n_{\mathrm{H}_{2}} \propto R^{-1.8}$. Inside $0.5 \mathrm{pc}$ from the center, instead, the gas is much more inhomogeneous and concentrated in a few high-density cores surrounding the (proto)stars. Our findings indicate that a self-regulating formation mechanism for the high-mass stars in G24.78 is plausible: in the proposed scenario star formation would occur from inside-out collapse of the parsec-scale clump, followed by infall reversal due to outflows powered by the newly formed massive stars. We also find that one of the two bipolar outflows powered by the embedded YSOs is more extended and hence older than the other, thus confirming the evolutionary sequence proposed in our previous article.
\end{abstract}

Key words. stars: formation - radio lines: ISM - ISM: individual objects: G24.78+0.08 - ISM: molecules

\section{Introduction}

How do stars form? Giving an answer to this question is an ambitious goal which requires profound knowledge of both the initial conditions and physical mechanisms involved in the process leading from a low density, cold, large scale cloud to the formation of a kernel hot and dense enough to ignite nuclear burning. In recent years much progress has been made in this field: satisfactory theoretical models describing the inside-out collapse of a single isothermal core have been elaborated and the subsequent pre-stellar evolution of the protostar has been studied in detail. Observations have taken profit of new powerful telescopes to investigate the interiors of molecular clouds at radio and infrared wavelengths, thus assessing their structure and stellar content. Although the picture is still very patchy, one may safely conclude that we have acquired a basic understanding of the formation process of stars with mass comparable to or less than that of the Sun. More massive stars instead remain a mystery: according to theory, stars above $8 M_{\odot}$ should "switch on" during the accretion phase (Palla \& Stahler 1993) thus halting or even reversing the infall by means of their radiation pressure. This leads to the paradox that O-B stars should not

Send offprint requests to: R. Cesaroni, e-mail: cesa@arcetri.astro.it exist. Possible ways to get round this problem are large accretion rates, accretion through disks, or formation from merging of lower mass stars (Stahler et al. 2000). Discriminating between these possibilities is a challenging observational task mostly for a reason: massive stars form in rich clusters emitting copious amounts of ionising photons that profoundly alter the surrounding environment. This makes it very difficult to find out the primordial configuration of the molecular cloud which represents the initial conditions for star formation. In conclusion, observational studies of massive star forming regions must privilege molecular clouds containing young stellar objects (YSOs) still in a very early phase of their evolution: in this case their impact on the surrounding material should be quite limited, thus making it possible to compare the properties of the parental cloud with those of the newly formed (proto)stars.

Many authors have studied high-mass star forming regions using a variety of targets, which are believed to be signposts of newly formed O-B stars (luminous IRAS point sources, ultracompact HII regions, etc.). Among these, Codella et al. (1997) performed a survey towards a sample of $\mathrm{H}_{2} \mathrm{O}$ and $\mathrm{OH}$ maser sources in the attempt to assess the presence of molecular cores associated with the maser spots. The main result is that in all cases a warm dense core is found coincident 
with the masers. In this article we will consider the case of $\mathrm{G} 24.78+0.08$ a source located at a distance of $7.7 \mathrm{kpc}$ (Forster \& Caswell 1989), which stands unique for its properties. Two groups of maser spots are found, one with only $\mathrm{H}_{2} \mathrm{O}$ emission, the other with both types of masers. The latter group is associated with an unresolved ultracompact (UC) HII region and with a hot molecular core, whereas the former seems embedded in a colder core and no free-free continuum is detected towards it. Moreover, slightly offset from the $\mathrm{H}_{2} \mathrm{O}$ and $\mathrm{OH}$ masers another UC HII region is seen, which looks more extended and apparently not associated with any high density peak of the molecular gas. Codella et al. (1997) conclude that they might be observing three high-mass YSOs in different evolutionary phases. Interestingly, in all groups the maser spots are distributed over $\sim 1^{\prime \prime}$, along parallel directions: since $\mathrm{H}_{2} \mathrm{O}$ masers are believed to be closely associated with outflows (see e.g. Felli et al. 1992; Moscadelli et al. 2000), Codella et al. (1997) suggest that the maser spots might trace the axes of putative outflows from the embedded YSOs.

Recently, follow-up observations by Furuya et al. (2002; hereafter Paper I) have fully confirmed the previous hypotheses. In particular, two bipolar outflows have been found: as expected, these are centred on the masers and aligned parallel to the distributions of the spots. Also, a new molecular core has been detected, which brings to 4 the number of putative high-mass YSOs in the region. In Paper I we argued that the newly detected core might host a massive protostar. The basic conclusion is that $\mathrm{G} 24.78$ consists of a cluster of very young massive (proto)stars in different evolutionary phases, but still deeply embedded in their parental cloud. This region represents an ideal laboratory for studying the structure of a highmass star-forming cloud and the feedback between this and the embedded YSOs.

With this in mind, we have decided to carry out observations of G24.78 on a scale of a few parsecs, zooming out from the relatively small region $(\sim 0.3 \mathrm{pc})$ previously mapped. In this article we present the results of single-dish observations of the molecular cloud associated with G24.78. We also illustrate in better detail the findings of the interferometric observations of Paper I. The combination of low and high angular resolution observations make it possible a thorough analysis of the gas distribution and physical parameters, and allow us to study the interaction between the newly formed stars and their natal environment.

\section{Observations and data reduction}

\subsection{IRAM Plateau de Bure interferometer}

The $2.6 \mathrm{~mm}$ observations were carried out with the IRAM $^{1}$ 5-antenna interferometer in April and May 1998. The D and $\mathrm{C} 1$ configurations were used, with baselines up to $147 \mathrm{~m}$. The phase tracking centre was $\alpha(\mathrm{J} 2000)=18^{\mathrm{h}} 36^{\mathrm{m}} 13^{\mathrm{s}} .10$ $\delta(\mathrm{J} 2000)=-07^{\circ} 12^{\prime} 08^{\prime \prime}$. 0 . The $82-116 \mathrm{GHz}$ SIS receiver was tuned single side-band at the ${ }^{12} \mathrm{CO} J=1-0$ frequency $(115.271 \mathrm{GHz})$. The facility correlator was configured with a

\footnotetext{
${ }^{1}$ IRAM is supported by INSU/CNRS (France), MPG (Germany) and IGN (Spain).
}

bandwidth of $40 \mathrm{MHz}$ centered on the ${ }^{12} \mathrm{CO}$ line, while two bandwidths of $160 \mathrm{MHz}$ each were combined to measure the continuum. Typical system temperatures were about $150 \mathrm{~K}$. The clean beam was 5.'3 $\times$ 4.' $1\left(\mathrm{PA}-162^{\circ}\right)$. Amplitude calibration was achieved by observing $3 \mathrm{C} 273$, which was assumed to have a flux of $16.7 \mathrm{Jy}$, while 1830-210 (1.7 Jy) and 1741038 (3.0 Jy) were used as phase calibrators. The typical RMS phase noise on the longest baselines was $35^{\circ}$. Calibration and data reduction were done using the GILDAS software developed at IRAM and Observatoire de Grenoble. Continuum subtraction was performed in the $(u, v)$ plane by using the integral over the line-free channels of the $160 \mathrm{MHz}$ units. Finally, channel maps were produced for the ${ }^{12} \mathrm{CO} J=1-0$ line by averaging the observed $156 \mathrm{kHz}$ channel spacing spectra up to a final value of $625 \mathrm{kHz}$, corresponding to $1.63 \mathrm{~km} \mathrm{~s}^{-1}$ at $2.6 \mathrm{~mm}$. The resulting clean beam is $5{ }^{\prime \prime} 3 \times 4^{\prime \prime} .1\left(\mathrm{PA}-162^{\circ}\right)$ and the conversion factor from flux to brightness temperature in the synthesised beam is $\sim 23.7 \mathrm{Jy} \mathrm{K}^{-1}$.

\subsection{Nobeyama Millimeter Array}

Interferometric observations of molecular lines and continuum emission at 2-mm band were carried out using the sixelement Nobeyama Millimeter Array (NMA) at Nobeyama Radio Observatory ${ }^{2}$ from December 2000 to January 2002 with three array configurations. In the most expanded configuration, the Nobeyama 45-m telescope was added as an element antenna of the interferometer: the telescope is located close to the end of the North-South arm of the NMA. Resulting projected baselines ranged from 4.1 to $198 \mathrm{k} \lambda$ and the synthesized beam size with natural weighting $2.3^{\prime \prime} \times 1.5^{\prime \prime}$. Our observations will miss $50 \%$ of the flux from structures $\gtrsim 15^{\prime \prime}$ (see Wilner \& Welch 1994), corresponding to $\sim 0.6 \mathrm{pc}$ at a distance of $7.7 \mathrm{kpc}$. The phase tracking centre was $\alpha(\mathrm{B} 1950)=18^{\mathrm{h}} 33^{\mathrm{m}} 30^{\mathrm{s}} 50 \delta(\mathrm{B} 1950)=-07^{\circ} 14^{\prime} 42^{\prime \prime}$. 0 . Both the NMA antennas and $45-\mathrm{m}$ telescope are equipped with SIS receivers having system noise temperatures in double-sideband of $300-500 \mathrm{~K}$ toward the zenith at $147 \mathrm{GHz}$. We checked the telescope pointing of the 45-m telescope every 90-120 min by five-point mapping of the $\mathrm{SiO} J=1-0$ maser line at $43 \mathrm{GHz}$ from IRC +00363 . The relative pointing offset between the 40 and $150 \mathrm{GHz}$ SIS receivers was less than $2^{\prime \prime}$ and the pointing error was within $3^{\prime \prime}$ during the observations.

We tuned the $150 \mathrm{GHz}$ SIS receiver at the frequency of $C^{32} S J=3-2$ line $(146.969047 \mathrm{GHz})$ and used two types of backends: the digital spectrocorrelator, FX, and the XFtype correlator, i.e. the Ultra Wide Band Correlator (UWBC; Okumura et al. 2000). The FX correlator was configured with 1024 channels per baseline and a bandwidth of $32 \mathrm{MHz}$, giving a velocity resolution of $0.0625 \mathrm{~km} \mathrm{~s}^{-1}$ with a velocity coverage of $64 \mathrm{~km} \mathrm{~s}^{-1}$. To cover not only the $\mathrm{C}^{32} \mathrm{~S}$ line but also all $K$ components of the $\mathrm{CH}_{3} \mathrm{CN} J=8-7$ transition, the UWBC was configured in the $512 \mathrm{MHz}$ bandwidth mode with 256 channels, providing a velocity resolution of

\footnotetext{
2 The Nobeyama Radio Observatory is a branch of the National Astronomical Observatory, operated by the Ministry of Education, Culture, Sports, Science and Technology Japan.
} 
$8.0 \mathrm{~km} \mathrm{~s}^{-1}$ after on-line smoothing. We used 3C 279 as bandpass calibrator and 1741-038 as phase and gain calibrator for the observations. From observations of Uranus, the flux density of 1741-038 was determined to be $2.5 \mathrm{Jy}$ during the observation period. The overall flux uncertainty is about $20 \%$. The data calibration was done using the UVPROCII software developed at the NRO and the image construction was performed using the AIPS package of NRAO. The typical $1 \sigma$ RMS noise levels were $120 \mathrm{mJy}^{2}$ beam $^{-1}$, after smoothing to $0.5 \mathrm{~km} \mathrm{~s}^{-1}$ resolution, for the $\mathrm{C}^{32} \mathrm{~S} J=3-2$ line, $48 \mathrm{mJy}$ beam ${ }^{-1}$ with $8.0 \mathrm{~km} \mathrm{~s}^{-1}$ resolution for the $\mathrm{CH}_{3} \mathrm{CN}$ lines, and $2.5 \mathrm{mJy}^{-1}$ beam $^{-1}$ for the $147 \mathrm{GHz}$ continuum emission which used an effective bandwidth of $\sim 800 \mathrm{MHz}$, after merging the line free channels of the signal band with those from the image side band at $134 \mathrm{GHz}$.

\subsection{Very Large Array}

The G24.78 region was observed with the $\mathrm{NRAO}^{3}$ Very Large Array (VLA) in its most compact (D) configuration on December 29, 2001. The phase tracking centre was $\alpha(\mathrm{J} 2000)=18^{\mathrm{h}} 36^{\mathrm{m}} 12^{\mathrm{s}} .66 \delta(\mathrm{J} 2000)=-07^{\circ} 12^{\prime} 10^{\prime} \cdot 146$. The source was observed in the $Q$-band $(43.34 \mathrm{GHz}, 0.7 \mathrm{~cm})$, at the time of the observations 25 antennas with $Q$-band receivers were available. We used an observing cycle with total duration of $5 \mathrm{~min}$ and an efficiency of $\sim 60 \%$. Hourly pointing at X-band on the calibrators were used to correct for pointing drifts during the $Q$-band observations. The flux density scale was set by observing 3C286 and 3C48, while 1832-105 (0.7 Jy) was used as a phase calibrator. The flux calibration is expected to be accurate within $20 \%$. All data editing and calibration has been performed using standard tasks within the NRAO AIPS software package. Two self-calibration (phase only) iterations were performed to improve the dynamic range of the final maps. All images presented here have been produced using the AIPS IMAGR task with robust weighting of the $(u, v)$ datasets the resulting synthesized $H P B W$ is 2 "' $3 \times 11^{\prime \prime} 7$. No correction for primary beam attenuation has been applied.

\subsection{IRAM 30-m telescope}

The observations with the IRAM 30-m telescope at Pico Veleta (Granada, Spain) were carried out in September 2001. Table 1 summarises the observed molecular species, transitions, rest frequencies, half power beam widths $(H P B W \mathrm{~s})$, and typical system temperature $\left(T_{\text {sys }}\right)$ expressed in main-beam brightness temperature $\left(T_{\mathrm{MB}}\right)$ units. The main beam efficiency varies from about 0.8 (at $96 \mathrm{GHz}$ ) to 0.5 (at $258 \mathrm{GHz}$ ). The pointing was checked about every hour by observing planets and nearby continuum sources and it was found to be accurate to within $4^{\prime \prime}$. High spectral resolution $(80 \mathrm{kHz})$ data have been obtained with an autocorrelator (AC) spectrometer split into four parts to allow simultaneous observations of four different transitions as well as with a $100 \mathrm{kHz}$ filter bank. Moreover, lower resolution spectra have been obtained by

\footnotetext{
3 The National Radio Astronomy Observatory is a facility of the National Science Foundation operated under cooperative agreement by Associated Universities, Inc.
}

Table 1. List of molecular species, transitions, and observational parameters of the data collected with the 30-m IRAM telescope.

\begin{tabular}{lrrr}
\hline \hline Transition & $\begin{array}{c}\text { Frequency }\left(^{a}\right) \\
(\mathrm{MHz})\end{array}$ & $\begin{array}{c}H P B W \\
\left({ }^{\prime \prime}\right)\end{array}$ & $\begin{array}{c}T_{\text {sys }} \\
(\mathrm{K})\end{array}$ \\
\hline $\mathrm{C}^{34} \mathrm{~S} \quad J=2-1$ & 96412.982 & 25 & 250 \\
$\mathrm{C}^{18} \mathrm{O} \quad J=1-0$ & 109782.156 & 22 & 240 \\
${ }^{13} \mathrm{CO} \quad J=1-0$ & 110201.352 & 22 & 200 \\
$\mathrm{CH}_{3} \mathrm{CN} \quad J_{\mathrm{K}}=66_{\mathrm{K}}-5_{\mathrm{K}}$ & 110383.508 & 22 & 350 \\
${ }^{12} \mathrm{CO} \quad J=1-0$ & 115271.195 & 21 & 480 \\
$\mathrm{C}^{34} \mathrm{~S} \quad J=3-2$ & 144617.147 & 17 & 500 \\
$\mathrm{C}^{32} \mathrm{~S} \quad J=3-2$ & 146969.047 & 17 & 390 \\
$\mathrm{CH}_{3} \mathrm{CN} \quad J_{\mathrm{K}}=8_{\mathrm{K}}-7_{\mathrm{K}}$ & 147174.594 & 17 & 740 \\
${ }^{13} \mathrm{CO} \quad J=2-1$ & 220398.688 & 11 & 800 \\
$\mathrm{CH}_{3} \mathrm{CN} \quad J_{\mathrm{K}}=12_{\mathrm{K}}-11_{\mathrm{K}}$ & 220747.266 & 11 & 2000 \\
${ }^{12} \mathrm{CO} \quad J=2-1$ & 230537.984 & 11 & 1500 \\
$\mathrm{C}^{34} \mathrm{~S} \quad J=5-4$ & 241016.176 & 10 & 2200 \\
$\mathrm{CH}_{3} \mathrm{CN} \quad J_{\mathrm{K}}=14_{\mathrm{K}}-13_{\mathrm{K}}$ & 257527.375 & 10 & 3900 \\
\hline
\end{tabular}

${ }^{a}$ ) for $\mathrm{CH}_{3} \mathrm{CN}$ the frequency of the $K=0$ transition is given.

using a $1 \mathrm{MHz}$ filter bank, split into a suitable number of parts. The receivers were tuned according to the following frequency setups which allowed us to observe several lines simultaneously: (i) ${ }^{12} \mathrm{CO}(1-0)$ and ${ }^{12} \mathrm{CO}(2-1)$; (ii) ${ }^{13} \mathrm{CO}(1-0), \mathrm{C}^{18} \mathrm{O}(1-$ $0), \mathrm{C}^{32} \mathrm{~S}(3-2)$, and ${ }^{13} \mathrm{CO}(2-1)$; (iii) $\mathrm{C}^{34} \mathrm{~S}(2-1)$, (3-2), and (54); and (iv) $\mathrm{CH}_{3} \mathrm{CN}(6-5),(8-7),(12-11)$, and (14-13). We stress that for the ${ }^{12} \mathrm{CO}$ lines and for the $\mathrm{C}^{34} \mathrm{~S}(5-4)$ transition two receivers have been used simultaneously in order to improve the $S / N$ ratio.

The ${ }^{12} \mathrm{CO}$ and ${ }^{13} \mathrm{CO}(1-0)$ data were collected with observations in on-the-fly mode, dumping spectra every $4^{\prime \prime}$ or $2 \mathrm{~s}$. Off-source spectra were observed every three on-source subscans. The off-source position was displaced by $+7071^{\prime \prime}$ in RA and $-947^{\prime \prime}$ in Dec, and was checked for absence of ${ }^{12} \mathrm{CO}$ emission by means of frequency switching observations. We observed the same region alternatively scanning along the RA and Dec directions (B1950 coordinates were used), in order to build up more homogeneous maps. The $\mathrm{CH}_{3} \mathrm{CN}$ and $\mathrm{C}^{34} \mathrm{~S}$ observations were performed using the wobbler switching mode with a $240^{\prime \prime}$ beam-throw and a phase-duration of $2 \mathrm{~s}$. The spectra were calibrated with the standard chopper wheel method and are reported here in $T_{\mathrm{MB}}$ units. The data reduction were done by using the standard GILDAS software.

In order to improve the absolute positions of the $\mathrm{C}^{34} \mathrm{~S}$ and $\mathrm{C}^{32} \mathrm{~S}(3-2)$ maps we realigned them to interferometric maps obtained in the same lines. For the $\mathrm{C}^{34} \mathrm{~S}$ maps we have used a map obtained with the BIMA interferometer in the $\mathrm{C}^{34} \mathrm{~S}(2-1)$ line (Beltrán, priv. communication): the 30-m maps have been shifted by $\left(-1{ }^{\prime \prime} 5,-3{ }^{\prime \prime} 5\right)$. For $\mathrm{C}^{32} \mathrm{~S}(3-2)$ instead, the NMA map has been used as a reference: and the two single dish maps have been shifted by $\left(+1\right.$.' $^{\prime},+3$.' $\left.^{\prime} 1\right)$ and $\left(-1\right.$.' $^{\prime} 1,-2$.' $\left.^{\prime}\right)$, respectively. We note that all the corrections applied are within the 30-m pointing error. 


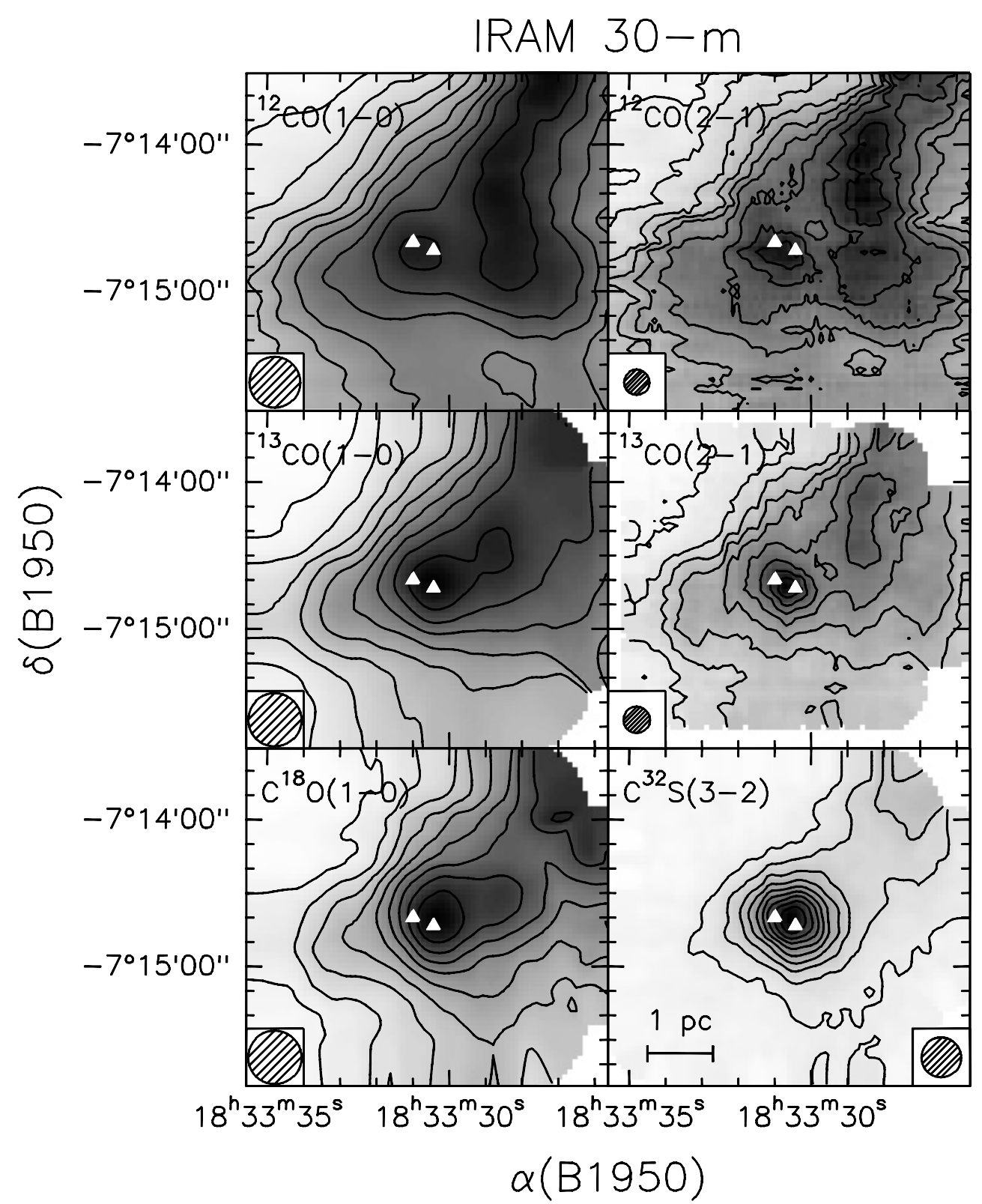

Fig. 1. Maps of the emission integrated under the whole line profile for six rotational transitions observed with the 30-m telescope. Contour levels range from 10 to $90 \%$ in steps of $10 \%$ of the peak intensity. In all cases the lowest contour is above the $3 \sigma$ limit of the corresponding map. The white triangles mark the positions of the $\mathrm{H}_{2} \mathrm{O}$ maser spots (Forster \& Caswell 1999). The telescope $H P B W$ is drawn as a shaded circle in the bottom of each map.

\section{Results}

In this section we present the maps obtained with the IRAM 30-m telescope. We also illustrate the results of interferometric observations performed with the PdBI and NMA, part of which has been shown in Paper I: here, we will present additional maps and perform a comparison between single-dish and interferometric data in the same tracers.

\subsection{Single-dish data}

\subsubsection{Line}

We have used the IRAM 30-m telescope to map the molecular cloud associated with the high-mass YSOs identified in Paper I.
We have observed six rotational transitions of four molecular species over a region $\sim 2^{\prime}$ in size. The maps of the integrated line emission are shown in Fig. 1: here the locations of the two groups of $\mathrm{H}_{2} \mathrm{O}$ masers are shown to pin point the position of the (proto) stellar cluster found in Paper I. In all tracers the emission peaks towards the masers, but the global pattern is quite different depending on the transition. A comparison between the $\mathrm{CO}$ isotopomers is illuminating under this respect: while the central clump associated with the masers is clearly seen in all lines, the elongated structure traced by the ${ }^{12} \mathrm{CO}$ transitions to the west of the main peak gets fainter when imaged in ${ }^{13} \mathrm{CO}$ and $\mathrm{C}^{18} \mathrm{O}$, and is hardly visible in the $\mathrm{C}^{32} \mathrm{~S}$ map. Since the latter is a high-density tracer, these results demonstrate that the elongated structure is characterised by relatively large column density, but low volume density. Such a conclusion is reinforced by 
the distribution of the CO lines optical depth, which we shall derive in Sect. 4.2: here, we anticipate that the elongated structure does not coincide with a clear peak in the optical depth map (cf. the white dotted contour to the west in Fig. 15), but with a region where the optical depth is slightly enhanced.

In conclusion, we believe that our maps demonstrate the existence of a well defined clump, coincident with the YSOs of interest for us. Hereafter we shall use the word "clump" to indicate the middle-scale ( $\sim 1 \mathrm{pc}$ ) cloud associated with the G24.78 cluster, not to be confused with the much smaller $(\sim 0.07 \mathrm{pc})$ "cores" imaged in Paper I.

Smaller maps in the $\mathrm{C}^{34} \mathrm{~S}(2-1),(3-2)$, and (5-4) lines have also been made. These are shown in Fig. 2, where the positions and names of the four YSOs identified in Paper I are reported. The $\mathrm{C}^{34} \mathrm{~S}$ emission peaks towards the position of core $\mathrm{A}$ and extends over a region including all YSOs. Given the low angular resolution $\left(\geq 10^{\prime \prime}\right)$, it is difficult to find out the contribution of the other YSOs to the $\mathrm{C}^{34} \mathrm{~S}$ emission, but it seems clear that the latter originates from a region of $\sim 15^{\prime \prime}$.

The complexity of the region under study is confirmed by the line profiles in Fig. 3. While the optically thin lines of $\mathrm{C}^{34} \mathrm{~S}$ and $\mathrm{C}^{18} \mathrm{O}$ have an almost Gaussian shape, the profiles of thicker transitions such as those of ${ }^{12} \mathrm{CO}$ and ${ }^{13} \mathrm{CO}$ present multiple peaks and dips. This is very likely due to the presence of velocity gradients in the molecular cloud and possibly to clouds lying along the line of sight but physically unrelated to the region of interest for us. We believe that also self-absorption may affect the line profiles. In fact, one can see that going from optically thin to thick tracers, the peak at $110.3 \mathrm{~km} \mathrm{~s}^{-1}$ (the systemic velocity of G24.78) is shifting towards higher velocities: this effect can be explained by blue-shifted self-absorption. In Sect. 4.1.2 it will be shown that this is indeed the case.

We have also observed four rotational transitions of $\mathrm{CH}_{3} \mathrm{CN}$ towards cores $\mathrm{A}$ and $\mathrm{C}-$ in Paper I no $\mathrm{CH}_{3} \mathrm{CN}$ emission was not detected from cores $\mathrm{B}$ and $\mathrm{D}$. The spectra are shown in Fig. 4, where also a comparison with the corresponding spectra obtained with the NMA is made (shaded profiles). No noticeable difference between the profiles of the two cores can be seen, although the intensity of the lines is stronger towards A. However, the $30-\mathrm{m} \mathrm{HPBW}$ is greater than or comparable to the angular separation between the two cores: this makes it very difficult to disentangle the contribution of one core from that of the other, especially for the (6-5) and (8-7) lines. Such an effect can be clearly seen in the (8-7) transitions by comparing the single-dish line profiles corresponding to $H P B W \simeq 17^{\prime \prime}$ with those obtained with the NMA, whose angular resolution is $\sim 2^{\prime \prime}$, i.e. much smaller than the separation between $\mathrm{A}$ and $\mathrm{C}$ : the single-dish profile towards $\mathrm{C}$ is obviously strongly "contaminated" by the emission arising from A.

It is worth pointing out that rotational diagrams of $\mathrm{CH}_{3} \mathrm{CN}$ could be used to estimate the temperatures of the cores, as done by various authors (see e.g. Olmi et al. 1993). In our case this method gives temperatures above $300 \mathrm{~K}$ for both cores. This is very likely the effect of large optical depth, as proved by the detection of some $\mathrm{CH}_{3}{ }^{13} \mathrm{CN}(8-7)$ transitions: for the $K=0$ and 1 lines the intensity ratio between isotopomer and main species gives an optical depth of $\sim 15$, assuming an abundance ratio of 72 between $\mathrm{CH}_{3} \mathrm{CN}$ and $\mathrm{CH}_{3}{ }^{13} \mathrm{CN}$ (Wilson \& Rood 1994).

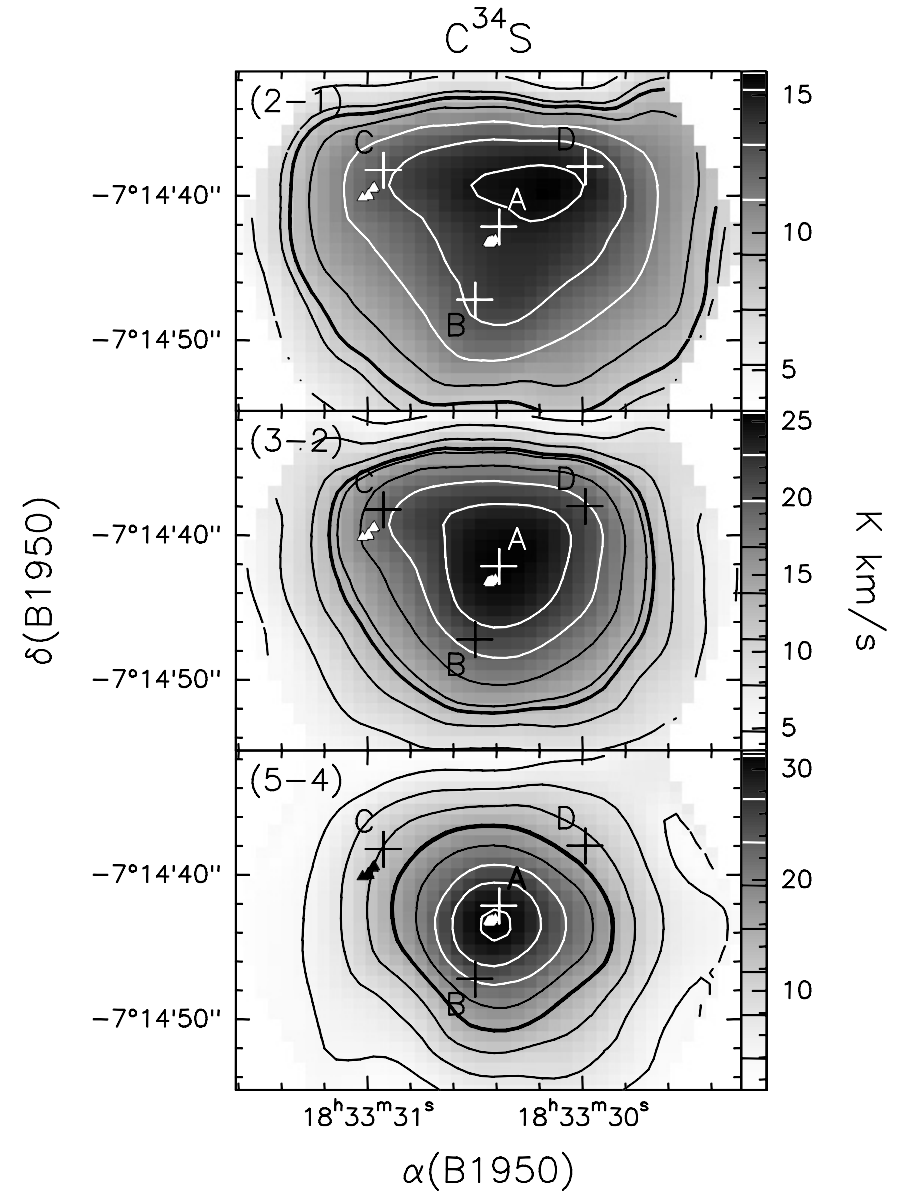

Fig. 2. IRAM 30-m maps of the $\mathrm{C}^{34} \mathrm{~S}(2-1)$, (3-2), and (5-4) emission integrated under the line from 105 to $116 \mathrm{~km} \mathrm{~s}^{-1}$. The contour levels are indicated in the wedge to the right of each panel. The thick contour corresponds to the $50 \%$ level. Triangles mark the positions of the $\mathrm{H}_{2} \mathrm{O}$ maser spots (Forster \& Caswell 1999), while the crosses and the corresponding labels identify the objects according to the notation used in Paper I.

In principle one could use the optically thin lines of the ${ }^{13} \mathrm{C}$ substituted species to get round the optical depth problem, but in our spectra only the $K=0$ and 1 components of $\mathrm{CH}_{3}{ }^{13} \mathrm{CN}$ are clearly detected, mostly the $K=0$ and 1: these are obviously insufficient to obtain an estimate of the rotational temperature.

In Table 2 we list the main parameters of the clump in G24.78 derived from the lines mapped by us. For each transition we give the full width at half maximum $\left(\Delta V_{\frac{1}{2}}\right)$ of the line profile, the full width at half power $(F W H P)$ of the clump, the corresponding angular $(\Theta)$ and linear $(D)$ diameters (obtained by deconvolution assuming source and beam to be Gaussian), the mass of the clump, the corresponding $\mathrm{H}_{2}$ volume density, and the virial mass computed from $D$ and $\Delta V_{\frac{1}{2}}$. The value of $\Delta V_{\frac{1}{2}}$ has been obtained by fitting a Gaussian to the line with the exception of the transitions whose profile is affected by overlap with other features and/or self-absorption: in these cases we have read off $\Delta V_{\frac{1}{2}}$ using the cursor. The masses have been calculated with different methods. In the case of the $\mathrm{CO}$ isotopomers, the emission has been integrated under the line 


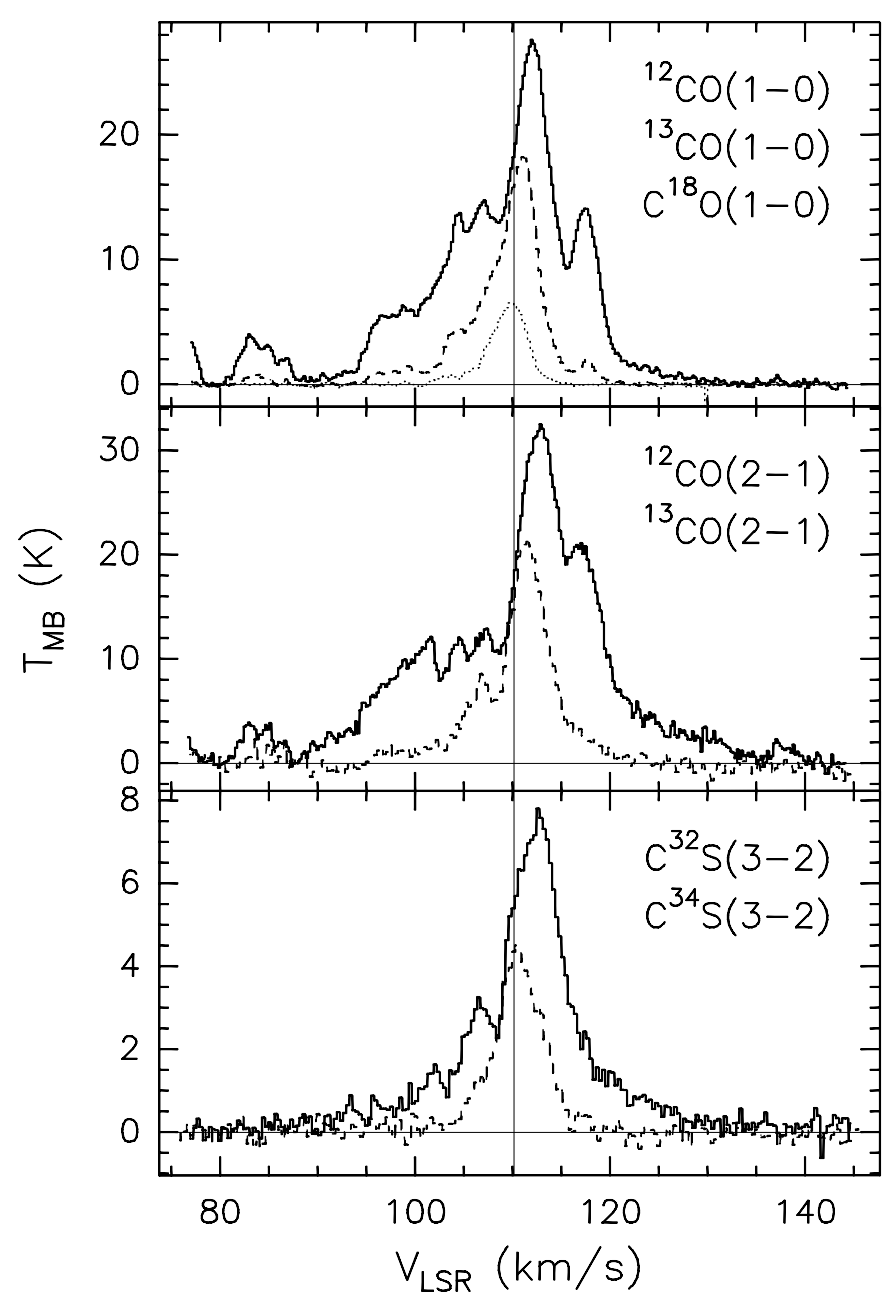

Fig. 3. Spectra observed towards the position of core A $(\alpha(\mathrm{B} 1950)=18: 33: 30.38 \delta(\mathrm{B} 1950)=-07: 14: 42.1)$. The full line corresponds to ${ }^{12} \mathrm{CO}(1-0),{ }^{12} \mathrm{CO}(2-1)$, and $\mathrm{C}^{32} \mathrm{~S}(3-2)$, the dashed line to ${ }^{13} \mathrm{CO}(1-0),{ }^{13} \mathrm{CO}(2-1)$, and $\mathrm{C}^{34} \mathrm{~S}(3-2)$ and the dotted line to $\mathrm{C}^{18} \mathrm{O}(1-0)$. The vertical thin line marks the systemic velocity of the G24.78 region, as given in Paper I.

and over the whole clump, and we have assumed LTE at a temperature of $30 \mathrm{~K}$ : the latter is equal to the maximum main beam brightness temperature measured with the 30-m telescope in the ${ }^{12} \mathrm{CO}(2-1)$ line. The values thus obtained have been corrected taking into account the line optical depth computed from the ratio between different isotopomers of the same species. The molecular abundances relative to $\mathrm{H}_{2}$ have been assumed equal to $8 \times 10^{-5}$ for ${ }^{12} \mathrm{CO}, 1.1 \times 10^{-6}$ for ${ }^{13} \mathrm{CO}, 1.4 \times 10^{-7}$ for $\mathrm{C}^{18} \mathrm{O}$, and $4 \times 10^{-10}$ for $\mathrm{C}^{34} \mathrm{~S}$ (Irvine et al. 1987; Wilson $\&$ Rood 1994). Mean densities have been derived in a trivial way assuming spherical symmetry. For $\mathrm{C}^{34} \mathrm{~S}$, instead, the value of the density has been derived directly from the line intensities, using the method described e.g. by Cesaroni et al. (1991) and from this the mass has been computed assuming the clump to be homogeneous and spherical. Finally, the mass pertaining to the $\mathrm{CH}_{3} \mathrm{CN}$ region has been taken equal to the sum of the masses of the cores detected in Paper I, because all the $\mathrm{CH}_{3} \mathrm{CN}$ emission arises from such cores (see Sect. 3.2).

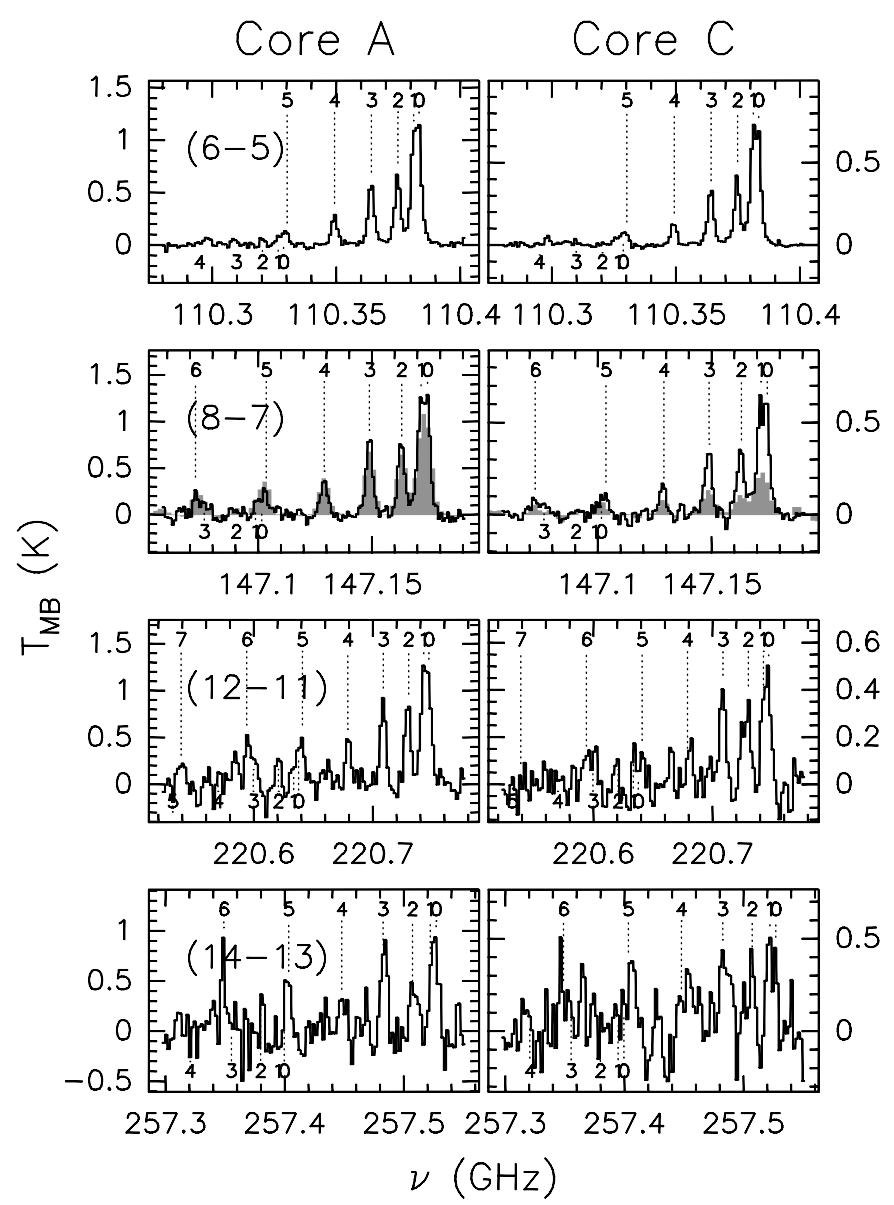

Fig. 4. Spectra of the methyl cyanide line emission towards cores A (left panels) and C (right panels). The notation of Paper I has been adopted for the core names. The vertical dotted lines indicate the positions of the different $K$ transitions of $\mathrm{CH}_{3} \mathrm{CN}$ (upper) and $\mathrm{CH}_{3}{ }^{13} \mathrm{CN}$ (lower). The shaded profiles are the spectra obtained by integrating the $\mathrm{CH}_{3} \mathrm{CN}(8-7)$ line emission observed with the NMA over cores A and $\mathrm{C}$.

Clearly, the mass and density values listed in Table 2 differ significantly one another. This result can be explained by the fact that different molecules trace regions with different densities: as a matter of fact, large gradients have been found in star forming clouds (see e.g. Fontani et al. 2002). However, some discrepancy exists even between mass estimates derived from lines of the same species, arising from approximately the same region: for instance, the masses obtained from ${ }^{12} \mathrm{CO}(1-$ 0 ) and ${ }^{12} \mathrm{CO}(2-1)$ differ by about a factor 3 . This could be due to a variety of effects. Very likely optical depths are large and hence difficult to estimate. In fact, the ratio between lines of isotopomers of the same species is prone to uncertainties related to the line profile: in our case, multiple components and self-absorption heavily affect the spectra, which in turn makes the line ratio quite unreliable. Furthermore, temperature gradients are likely to exist in the gas, which is not consistent with our assumption of isothermal clump. In conclusion, we believe that for the mass and density values in Table 2 an uncertainty of $\sim 3$ is in order.

Notwithstanding this caveat, it seems that the mass estimated from the molecular tracers is systematically greater than 
the corresponding virial mass: this is consistent with the conclusions of Fontani et al. (2002), who obtained the same result for a sample of clumps associated with high-mass YSOs. These findings raise the question of clump stability, which will be discussed in Sect. 5.

\subsubsection{Continuum}

Although no bolometric continuum observation of G24.78 has been performed by us, we used the line-free channels of the $\mathrm{CH}_{3} \mathrm{CN}$ spectra to obtain an estimate of the continuum level at the positions of cores $\mathrm{A}$ and $\mathrm{C}$. This method works reasonably well when a nutating secondary is used and if the weather conditions are stable. We have taken 5 spectra towards A and 11 spectra towards C: the flux estimate has been taken equal to the middle value between the minimum and the maximum fluxes measured at each position, while the error has been assumed equal to half the range between the minimum and the maximum. In Fig. 5 we compare the values of the fluxes thus obtained (big points) with the fits (curves) to the continuum measurements obtained by other means (small points). Details on the data and model fits have been given in Paper I. It must be noted that at 3 and $2 \mathrm{~mm}$ the $H P B W$ is much greater than the separation between $\mathrm{A}$ and $\mathrm{C}\left(\sim 8^{\prime \prime}\right)$, so that it is impossible to disentangle the contribution of each core to the total flux. To some extent this can be done at 1.4 and $1.2 \mathrm{~mm}$ where the angular resolution is $\sim 10^{\prime \prime}$, albeit with a large uncertainty especially on the flux of the fainter core (C). From Fig. 5 one can see that the total flux measured at 3 and $2 \mathrm{~mm}$ is very similar to the values obtained from interferometric observations, which supports the reliability of our single-dish estimates. However, the most interesting result is the good agreement between the two values measured at 1.4 and $1.2 \mathrm{~mm}$ and those expected on the basis of the model fit from Paper I. Such an agreement is especially evident in the top panel of Fig. 5, where the total flux from all cores is compared to the sum of the model fits of each core. In the bottom panel instead one can see that the $1 \mathrm{~mm}$ fluxes of core $\mathrm{C}$ lie above the corresponding fit. However, as explained above, it is difficult to estimate the flux of each single core: therefore, while the total flux is more accurate, the fluxes from $\mathrm{A}$ and $\mathrm{C}$ are prone to greater uncertainties. We conclude that our single-dish continuum measurements are consistent with the model fit presented in Paper I.

\subsection{Interferometric data}

In Paper I we have already illustrated the PdBI and NMA maps of the bipolar outflow in the ${ }^{12} \mathrm{CO}(1-0)$ line and of the cores in the $\mathrm{CH}_{3} \mathrm{CN}(8-7)$ line and in the millimeter continuum emission. Figure 6 shows the maps of the integrated emission in the ${ }^{12} \mathrm{CO}(1-0)$ and $\mathrm{C}^{32} \mathrm{~S}(3-2)$ lines: for the sake of comparison, we also plot the positions of the four objects identified in Paper I. We note that, while the $\mathrm{C}^{32} \mathrm{~S}$ emission clearly outlines cores $\mathrm{A}$ and $\mathrm{C}$, the main ${ }^{12} \mathrm{CO}$ peak is not perfectly coincident with the cores. We believe that such a disagreement is not real, but due to the complex structure of the ${ }^{12} \mathrm{CO}$ gas which cannot be properly mapped with the PdBI: this effect becomes dramatic

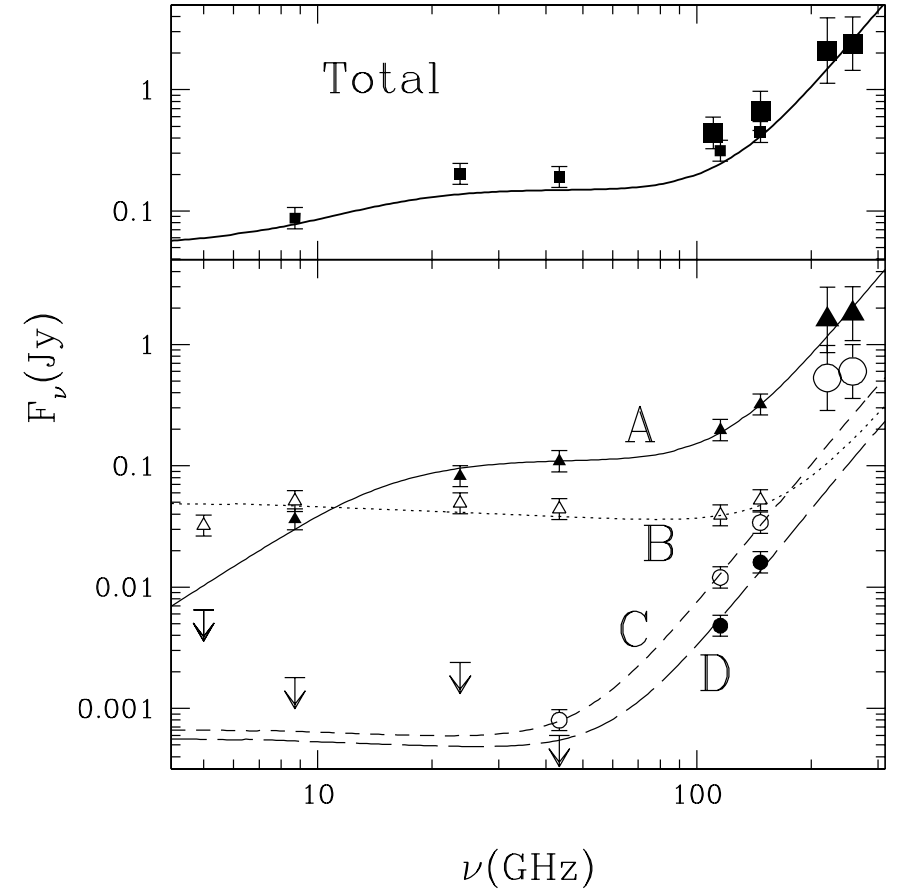

Fig. 5. Bottom panel: spectra of the continuum emission of the four objects identified in the G24.78 region. The letters identify each spectrum according to the notation of Paper I. The curves in the bottom panel are the model fits (see Paper I) to the interferometric measurements represented by the small points. The single-dish measurements toward cores A and C obtained in this study correspond instead to the bigger points. Note that the latter are not shown at 110 and $147 \mathrm{GHz}$ because it is impossible to disentangle the contribution of cores A and C. Top panel: spectrum of the total continuum flux from all sources. The curve is the sum of the model fits in the bottom panel. The bigger points indicate our single-dish measurements.

close to the line peak, where the emission is most extended and hence difficult to image with an interferometer. This effect will be better illustrated below (see Fig. 9). Another noticeable feature is the absence of emission towards D. This result has already been discussed in Paper I: here we recall that this represents evidence in favour of $\mathrm{D}$ being depleted, as expected for a cold, dense protostellar core. Finally, strong emission is detected towards B, which supports the conclusion of Paper I that the YSO in B is more evolved - and hence no more enshrouded by dense material.

It is also worth noting that in the NMA observations a few lines of $\mathrm{CH}_{3} \mathrm{OCH}_{3}$ and $\mathrm{C}_{2} \mathrm{H}_{5} \mathrm{CN}$ have been serendipitously detected. These are seen towards cores $\mathrm{A}$ and $\mathrm{C}$, as illustrated in Fig. 7, which shows the spectra towards the peaks of these cores. Rare molecular species are indeed commonly found in hot molecular cores, as demonstrated e.g. by the line surveys in Orion (see e.g. Blake et al. 1996).

Figure 8 shows the spectra of the $\mathrm{CH}_{3} \mathrm{CN}(8-7)$ and $\mathrm{C}^{32} \mathrm{~S}(3-$ 2) lines taken with the IRAM 30-m telescope and with the NMA. The latter has been obtained by cleaning the NMA data with a restoring beam equal to that of the 30-m telescope. The 30-m spectrum has been resampled to the (lower) spectral resolution of the interferometer. Within the noise, all the $\mathrm{CH}_{3} \mathrm{CN}$ emission seen by the $30-\mathrm{m}$ telescope is recovered with 
Table 2. Parameters of the molecular clump mapped with the IRAM 30-m telescope. A distance of $7.7 \mathrm{kpc}$ has been assumed.

\begin{tabular}{|c|c|c|c|c|c|c|c|}
\hline Line & $\begin{array}{c}\Delta V_{\frac{1}{2}} \\
\left(\mathrm{~km} \mathrm{~s}^{-1}\right)\end{array}$ & $\begin{array}{l}F W H P \\
(\operatorname{arcsec})\end{array}$ & $\begin{array}{c}\Theta \\
(\operatorname{arcsec})\end{array}$ & $\begin{array}{c}D \\
(\mathrm{pc}) \\
\end{array}$ & $\begin{array}{r}M \\
\left(\mathrm{~cm}^{-3}\right) \\
\end{array}$ & $\begin{array}{c}n_{\mathrm{H}_{2}} \\
\left(M_{\odot}\right)\end{array}$ & $\begin{array}{r}M_{\text {vir }} \\
\left(M_{\odot}\right) \\
\end{array}$ \\
\hline${ }^{12} \mathrm{CO}(1-0)$ & $5.4\left(^{a}\right)$ & 68 & 65 & 2.4 & 14600 & $4.0 \times 10^{4}$ & 7400 \\
\hline${ }^{12} \mathrm{CO}(2-1)$ & $5.2\left({ }^{a}\right)$ & 60 & 59 & 2.2 & 5500 & $2.0 \times 10^{4}$ & 6300 \\
\hline${ }^{13} \mathrm{CO}(1-0)$ & $4.7\left(^{a}\right)$ & 48 & 43 & 1.6 & 17500 & $1.7 \times 10^{5}$ & 3700 \\
\hline${ }^{13} \mathrm{CO}(2-1)$ & $4.6\left(^{a}\right)$ & 32 & 30 & 1.1 & 5800 & $8.5 \times 10^{4}$ & 2500 \\
\hline $\mathrm{C}^{18} \mathrm{O}(1-0)$ & $4.4\left(^{b}\right)$ & 44 & 38 & 1.4 & 24900 & $3.4 \times 10^{5}$ & 2900 \\
\hline$C^{32} S(3-2)$ & $6.4\left(^{a}\right)$ & 24 & 18 & 0.67 & - & - & 2900 \\
\hline$C^{34} S(2-1)$ & $4.6\left(^{b}\right)$ & 27 & 11 & 0.41 & 2400 & $1.0 \times 10^{6}$ & 912 \\
\hline$C^{34} S(3-2)$ & $5.4\left(^{b}\right)$ & 21 & 13 & 0.49 & " & $"$ & 1500 \\
\hline$C^{34} S(5-4)$ & $5.3\left(\left(^{b}\right)\right.$ & 14 & 10 & 0.37 & " & $"$ & 1100 \\
\hline $\mathrm{CH}_{3} \mathrm{CN}(8-7)$ & $5.5\left(^{b}\right)$ & 17 & 6 & 0.22 & $900\left({ }^{c}\right)$ & $3.1 \times 10^{6}$ & 710 \\
\hline
\end{tabular}

$\left({ }^{a}\right)$ Line profile affected by blending and/or self-absorption: FWHM measured manually.

(b) FWHM from Gaussian fit to the line profile.

${ }^{c}$ ) Total mass of the cores computed from Table 1 of Paper I.

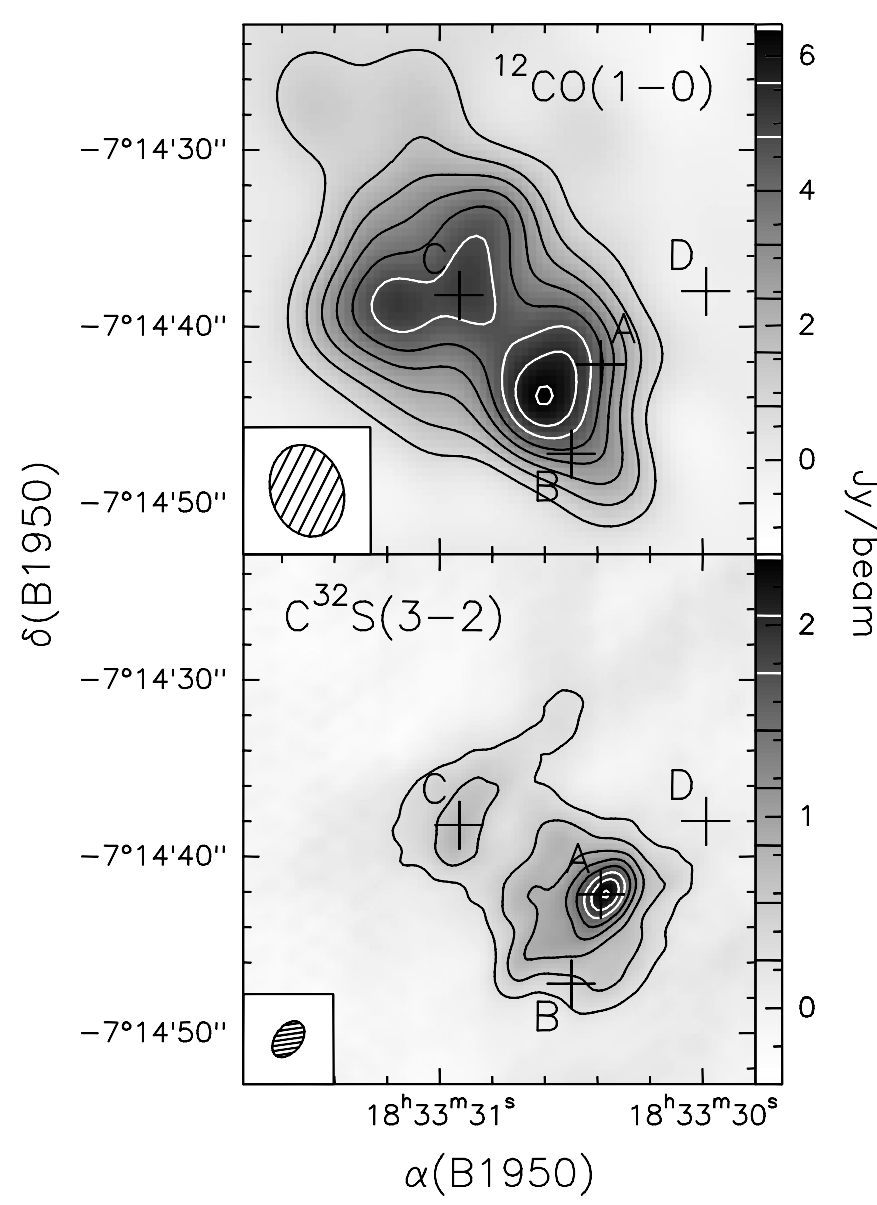

Fig. 6. PdBI (top panel) and NMA (bottom panel) maps obtained by integrating the emission under the ${ }^{12} \mathrm{CO}(1-0)$ and $\mathrm{C}^{32} \mathrm{~S}(3-2)$ lines, respectively. The crosses and corresponding labels mark the four objects identified by Paper I. Contour levels are drawn in the wedge beside each panel.

the NMA, which proves that the $\mathrm{CH}_{3} \mathrm{CN}$ lines originate entirely from the $\mathrm{A}$ and $\mathrm{C}$ cores identified in Paper I. On the contrary, a large fraction of the $\mathrm{C}^{32} \mathrm{~S}$ emission is not imaged in the core of the line. This is shown with better spectral resolution in the right panels of Fig. 9, where we also present a comparison between interferometric and single-dish maps obtained by integrating the $\mathrm{C}^{32} \mathrm{~S}(3-2)$ emission under the line. The left panels show the same thing for the ${ }^{12} \mathrm{CO}(1-0)$ transition observed with the 30-m telescope and the PdBI.

As expected, the interferometric maps filter out extended structures and are thus sensitive to the most compact, densest parts of the clump, i.e. cores A and C. The large scale emission seen with the 30-m telescope is instead completely resolved out by the PdBI and NMA. This effect is dramatic close to the systemic velocity $\left(\sim 110.3 \mathrm{~km} \mathrm{~s}^{-1}\right)$, where line emission is stronger and hence visible over more extended regions which cannot be imaged with our interferometric observations. On the contrary, the line wings are almost entirely recoverd. We conclude that the morphology and kinematics of the G24.78 cloud are very complex: that is why it is necessary to combine all the information available to analyse the structure of the molecular gas in this star forming region.

\section{Discussion}

In order to present an overview of the G24.78 region, we show in Fig. 10 a composite map where the ${ }^{12} \mathrm{CO}(1-0)$ line emission is superimposed to the $20 \mathrm{~cm}$ continuum image extracted from the NRAO VLA Sky Survey (NVSS; Condon et al. 1998). There is little doubt that we are dealing with a high-mass star forming region: to the north, one can see an extended Hir region traced by the free-free continuum emission, approximately coincident with a dip in a molecular ridge oriented north-south. The water masers (triangles) pin point the position of the cluster of massive YSOs studied in Paper I: these are located at the south-eastern end of the ridge. It seems that we are observing a filament of molecular gas undergoing different episodes of star formation, of which the one associated with the masers appears to be the most recent.

In the following, we shall use all the available multitracer, multi-scale maps of the $\mathrm{G} 24.78$ region to investigate the "anatomy" of the molecular gas over all scales ranging from $\sim 2$ pc to $\sim 0.1$ pc. 


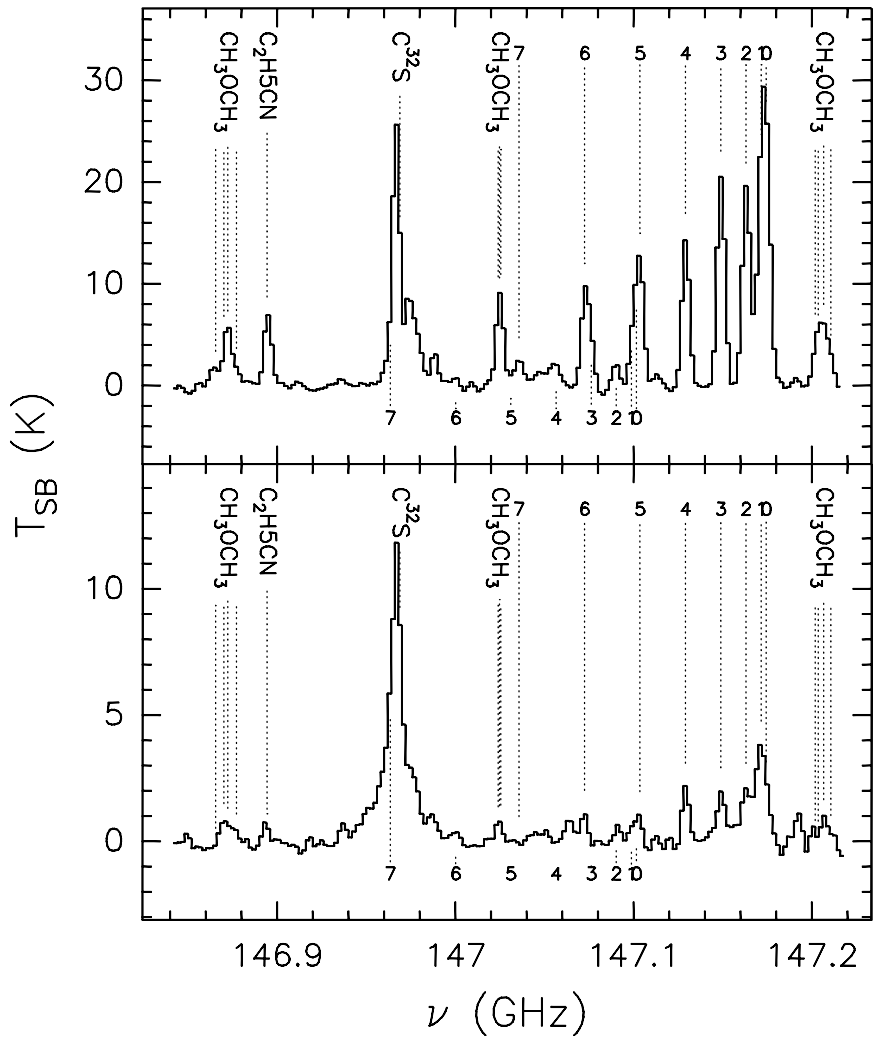

Fig. 7. Spectra towards the peak positions of cores A and C obtained with the NMA. The $y$-axis scale is expressed in brightness temperature in the synthesised beam of the interferometer. The dotted vertical lines and corresponding labels indicate the position of the $\mathrm{CH}_{3} \mathrm{CN}(8-$ 7), $\mathrm{CH}_{3}{ }^{13} \mathrm{CN}(8-7)$, and $\mathrm{C}^{32} \mathrm{~S}(3-2)$ lines; also indicated are the detected transitions of $\mathrm{CH}_{3} \mathrm{OCH}_{3}\left(5_{3,3}-5_{2,4}\right), \mathrm{CH}_{3} \mathrm{OCH}_{3}\left(7_{1,7}-6_{0,6}\right)$, and $\mathrm{C}_{2} \mathrm{H}_{5} \mathrm{CN}\left(17_{1,17}-16_{1,16}\right)$.

\subsection{The clump}

\subsubsection{Density gradient}

Massive YSOs are found embedded in dense molecular clumps a few parsecs in size. Recently, Fontani et al. (2002) have suggested that the clumps associated with high-mass YSOs might be on the verge of gravitational collapse. Their hypothesis is based on the fact that the masses of such clumps are systematically greater than the virial masses; also, they find density gradients of the type $n_{\mathrm{H}_{2}} \propto R^{-2.6}$, with $R$ distance from the clump centre, similar to those characterising singular isothermal spheres (Shu et al. 1987; Li 1999). We have seen in Sect. 3.1.1 that also in the case of G24.78 the mass of the clump is significantly greater than the corresponding virial mass. Now, we want to study the density structure of the clump. For this purpose, we plot in Fig. 11 the density estimated for each molecular tracer against the corresponding radius (see Table 2). Although the values of the masses derived from the $\mathrm{CO}$ isotopomers is quite uncertain for the reasons discussed above, the two quantities are well correlated (corr. coeff. 0.92) according to the relation $n_{\mathrm{H}_{2}} \propto R^{-1.8}$. Although flatter than that of Fontani et al. (2002), this curve confirms that the density gradient is steep.

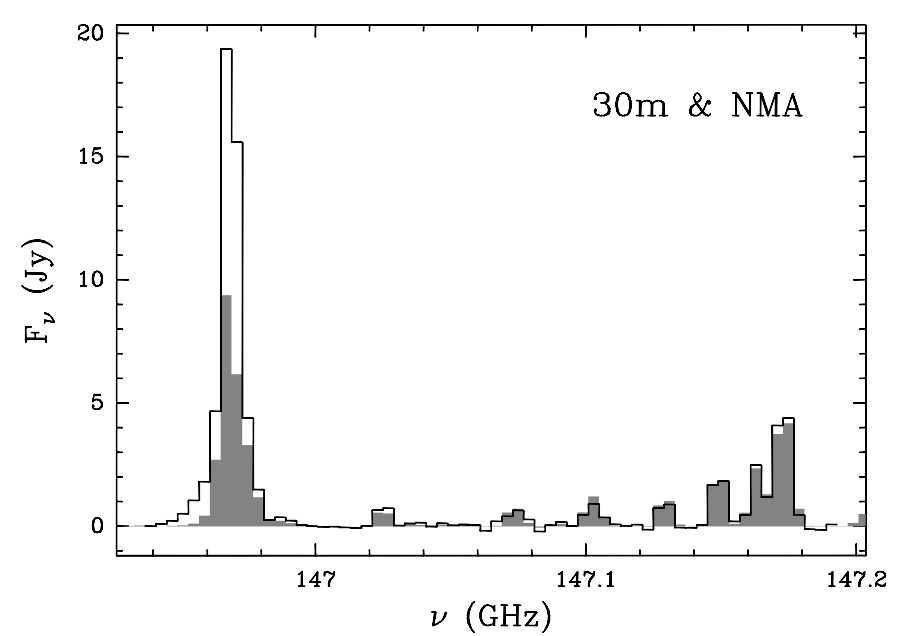

Fig. 8. Comparison between the $\mathrm{CH}_{3} \mathrm{CN}(8-7)$ and $\mathrm{C}^{32} \mathrm{~S}(3-2)$ spectra obtained with the IRAM 30-m telescope (full histogram) and NMA (shaded histogram) towards the position of core A. The two spectra have the same spectral and spatial resolution, as explained in the text.

Another way to study the dependence of density upon radius in the clump is to plot the column density, $N_{\mathrm{H}_{2}}$, obtained from an optically thin tracer such as $\mathrm{C}^{18} \mathrm{O}(1-0)$, as a function of distance from the clump centre. In order to obtain a mean value of $N_{\mathrm{H}_{2}}$ we have performed an azimuthal average of the $\mathrm{C}^{18} \mathrm{O}(1-0)$ line brightness along equally spaced annuli centred on the peak of the emission. Note that under the assumption of constant temperature and optically thin emission, $N_{\mathrm{H}_{2}}$ is proportional to the line brightness temperature, $T_{\mathrm{B}}$. In Fig. 12 we plot the main beam brightness temperature, $T_{\mathrm{MB}}$, normalised with respect to the peak value against the angular distance from the centre divided by the FWHP of the clump (44", according to Table 2). This profile has been fitted with a power law after convolution with the beam of the 30-m telescope. The best fit is obtained for $N_{\mathrm{H}_{2}} \propto R^{-0.8}$, which turns into a volume density $n_{\mathrm{H}_{2}} \propto R^{-1.8}$. This is consistent with the value derived above.

In conclusion, we believe that a density gradient as steep as $n_{\mathrm{H}_{2}} \propto R^{-1.8}$ represents a good description of the internal structure of the G24.78 clump. Theory (Shu et al. 1987) predicts an initial condition for star formation corresponding to a singular isothermal sphere with density $\propto R^{-2}$. This sphere should rapidly undergo inside-out collapse with the infalling gas having density $\propto R^{-1.5}$. Note that our slope is intermediate between these two values, which might suggest that, depending on the tracer, we are sampling both the static outer region with slope -2 and the collapsing inner region with slope -1.5 . In Sect. 5 we shall discuss this issue in better detail.

\subsubsection{Velocity field}

Beside density gradients, also temperature variations must exist inside the clump: in fact, Codella et al. (1997) measured temperatures as high as $90 \mathrm{~K}$ at $\sim 0.1 \mathrm{pc}$ from the centre, whereas we have measured $\sim 30 \mathrm{~K}$ in a region 10 times larger. These findings indicate that also the excitation temperature of some molecular species is likely to increase towards the centre of the clump: in this case, self-absorption should be seen in optically 


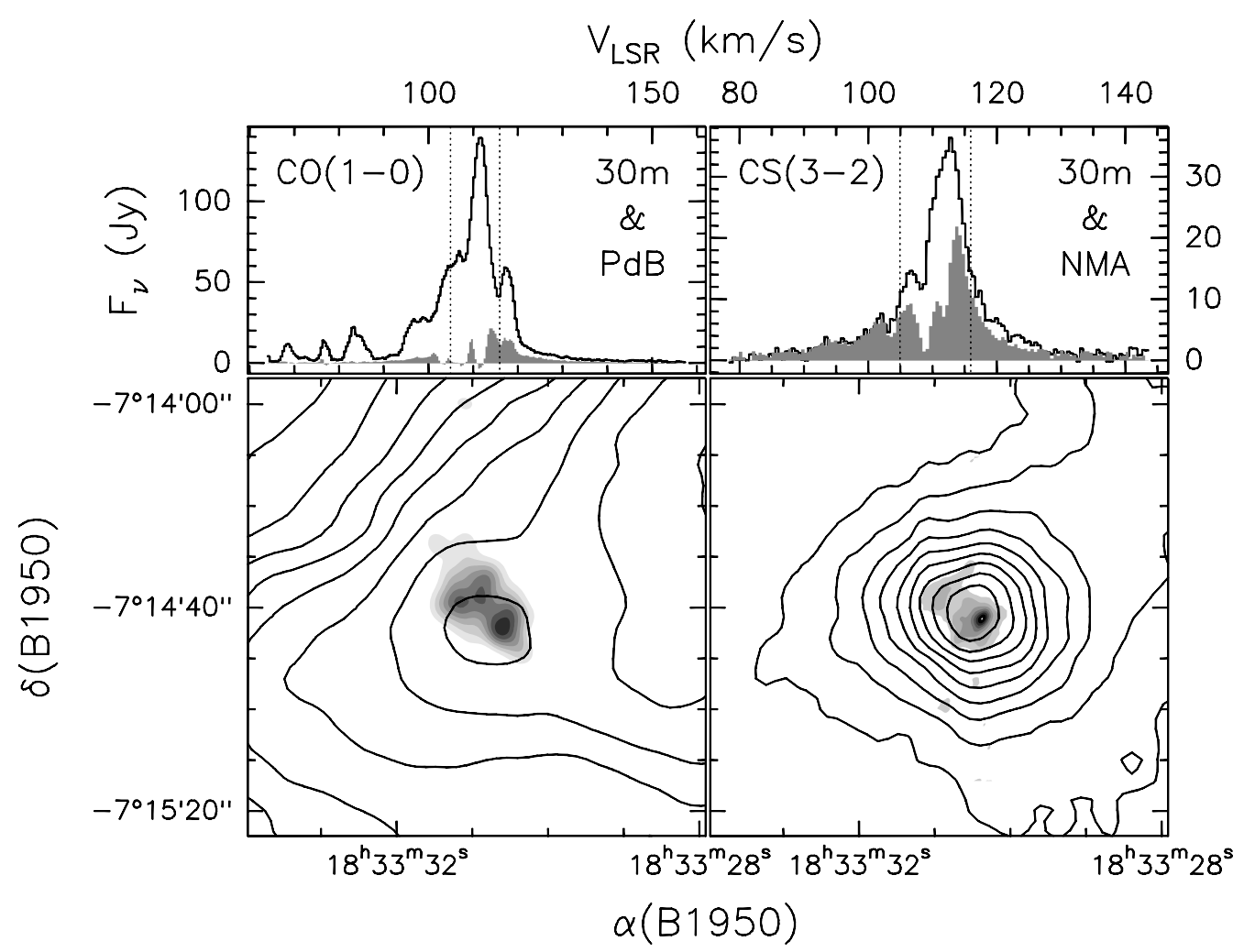

Fig. 9. Top panels: Same as Fig. 8 for the high spectral resolution spectra of the $\mathrm{C}^{32} \mathrm{~S}(3-2)$ (right panel) and ${ }^{12} \mathrm{CO}(1-0)($ left) lines. Bottom panels: Overlay of the single-dish (contours) and interferometer (grey scale) maps obtained by integrating the emission under the $\mathrm{C}^{32} \mathrm{~S}(3-2)$ (right) and ${ }^{12} \mathrm{CO}(1-0)$ (left) lines over the $V_{\mathrm{LSR}}$ range $105-116 \mathrm{~km} \mathrm{~s}^{-1}$. The latter velocities are marked with vertical dotted lines in the top panels.

thick lines. Moreover, if the optically thick gas is undergoing expansion or contraction, the absorption dip must be respectively blue- or red-shifted with respect to the systemic velocity. Indeed, this expectation seems confirmed by the profiles of the ${ }^{12} \mathrm{CO},{ }^{13} \mathrm{CO}$, and $\mathrm{C}^{32} \mathrm{~S}$ spectra of Fig. 3, which present dips at the peak velocity of more optically thin isotopomers such as $\mathrm{C}^{18} \mathrm{O}$ and $\mathrm{C}^{34} \mathrm{~S}$. In order to rule out the possibility that such profiles are due to overlap of multiple components, bf it is worth studying the spatial distribution of the $C^{32} S(3-2)$ line emission.

We computed mean profiles of the $\mathrm{C}^{32} \mathrm{~S}(3-2)$ transition by averaging the emission over circular annuli around the centre of the clump. The spectra thus obtained towards the centre and at radial offsets in multiples of the $H P B W$ are shown in Fig. 13. Approaching the border of the clump, the peak of the spectrum shifts towards the systemic velocity and the dip disappears, consistently with self-absorption being most effective along the line of sight through the centre, namely where the excitation temperature gradient is maximum. Furthermore, the dip is blue-shifted with respect to the systemic velocity, which indicates that the gas is expanding.

Another line of evidence for the existence of blue-shifted self-absorption can be obtained from the channel maps in the $\mathrm{C}^{32} \mathrm{~S}(3-2)$ line. Without blue- or red-shifted self-absorption the size measured in channels symmetrically displaced with respect to the systemic velocity should be the same; if such a self-absorption is present, instead, an asymmetry is introduced, so that the peak emission in channels affected by self-absorption must be depressed. The consequence of this effect is that the $F W H P$ measured in self-absorbed channels is larger; on the contrary, the size of the emission measured at a $3 \sigma$ level must be unaffected because it reflects just the maximum size of the clump at a given velocity. In order to verify these expectations, we have measured the $F W H P$ and the diameter at a $3 \sigma$ level (full width at zero intensity; hereafter $F W Z I$ ) in each channel of the $\mathrm{C}^{32} \mathrm{~S}(3-2)$ line. These are plotted in the bottom panel of Fig. 14: while the FWZI is approximately symmetric with respect to the systemic velocity, the $F W H P$ is by far larger in the blue-shifted channels and reaches its maximum in correspondence to the dip of the line profile.

We conclude that we are observing blue-shifted selfabsorption in the $\mathrm{C}^{32} \mathrm{~S}(3-2)$ line. The same result likely holds also for the optically thick CO isotopomers, but in this case it is more difficult to apply the same analysis to the data because of the presence of multiple spatial and spectral components. Our finding has a twofold consequence: it supports the existence of excitation temperature gradients (and hence density gradients) in the clump; and it proves that at least part of the clump is expanding. In the following, we shall investigate the origin of such an expansion by focusing our analysis on the region where the compact, star-forming cores studied in Paper I are located.

\subsection{The core region}

The structure and roundish appearance of the G24.78 clump indicate that on a large scale the gas is still unaffected by the 


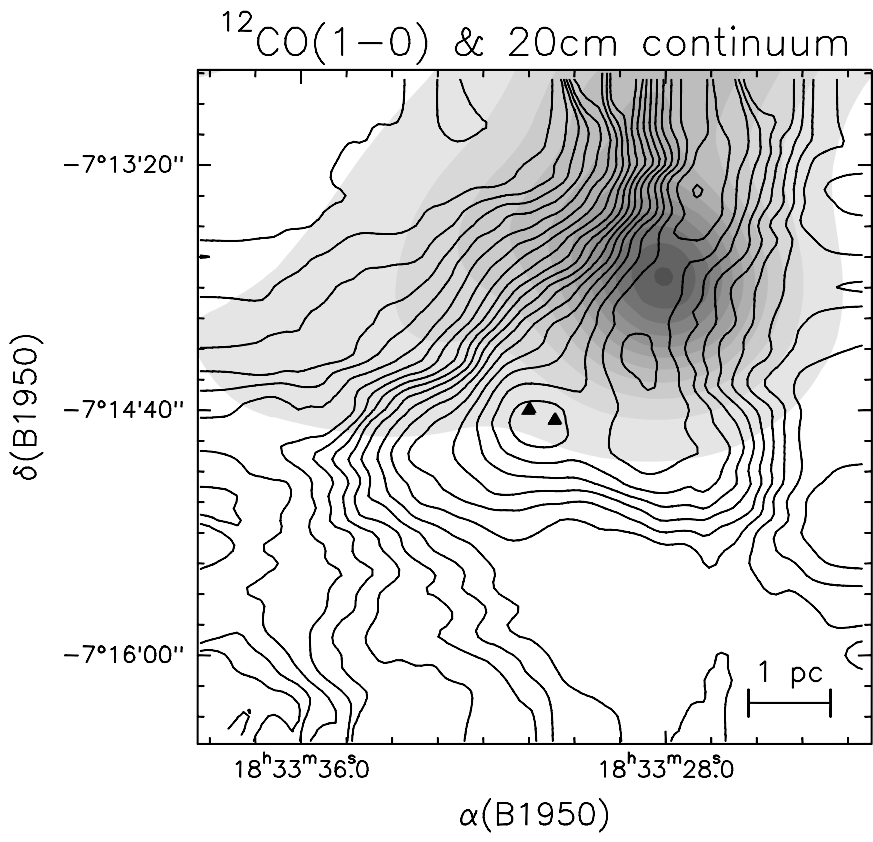

Fig. 10. Comparison between the ${ }^{12} \mathrm{CO}(1-0)$ map obtained by us with the IRAM 30-m telescope and the $20 \mathrm{~cm}$ continuum image extracted from the NVSS database. The ${ }^{12} \mathrm{CO}$ emission has been integrated under the line: contour levels range from 4 to 194 in steps of $10 \mathrm{~K} \mathrm{~km} \mathrm{~s}^{-1}$. The triangles mark the positions of the $\mathrm{H}_{2} \mathrm{O}$ maser spots identified by Forster \& Caswell (1999).

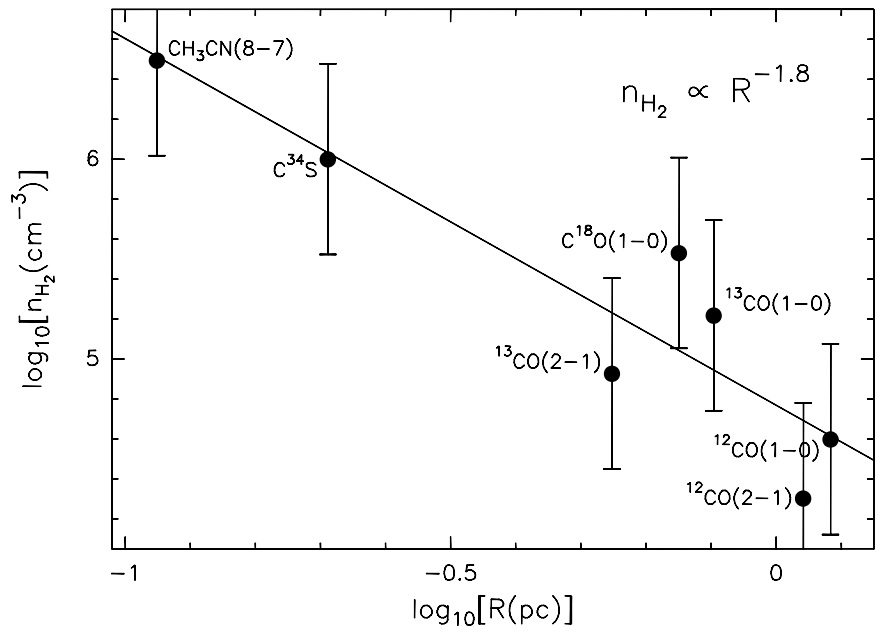

Fig. 11. Plot of $\mathrm{H}_{2}$ volume density versus clump radius for all the tracers observed by us. The values are taken from Table 2. An uncertainty of a factor 3 has been assumed according to the discussion in Sect. 3.1.1. The straight line is a linear fit to the data.

embedded YSOs. This implies that on $\sim 1$ pc one might be observing the "primordial" environment prior to the current star formation episode. The same conclusion cannot hold for the innermost regions, where the the gas has fragmented and condensed into dense, compact cores which contain newly born massive YSOs heavily interacting with the surrounding material.

In order to study the densest parts of the clump, one must use optically thin tracers such as $\mathrm{C}^{34} \mathrm{~S}$. This is demonstrated by Fig. 15, where a comparison is shown between the optical

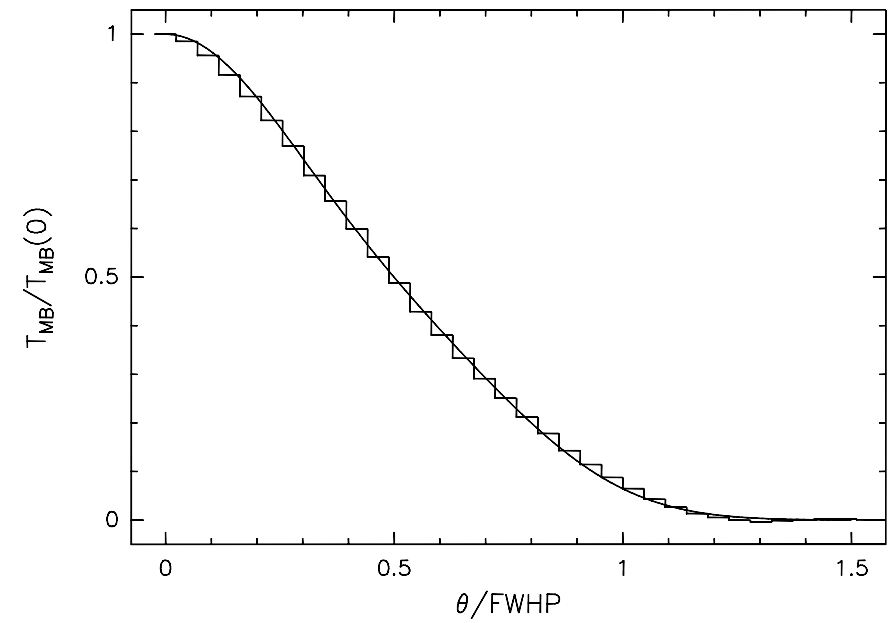

Fig. 12. The histogram is a plot of the mean main beam brightness temperature $\left(T_{\mathrm{MB}}\right)$ in the $\mathrm{C}^{18} \mathrm{O}(1-0)$ line versus the angular distance $(\theta)$ from the centre of the clump. Both quantities are normalised: the former with respect to the peak value, the latter with respect to the $F W H P$ of the clump. The mean has been obtained by averaging $T_{\mathrm{MB}}$ over equally spaced, concentric annuli. The full line is the best fit to the data of a power law convolved with the instrumental beam and corresponds to $T_{\mathrm{B}} \propto \theta^{-0.8}$.

depth distributions of the ${ }^{13} \mathrm{CO}(1-0),{ }^{13} \mathrm{CO}(2-1), \mathrm{C}^{18} \mathrm{O}(1-0)$ and $\mathrm{C}^{34} \mathrm{~S}(3-2)$ lines. The optical depth estimates have been obtained from the ratios between ${ }^{12} \mathrm{CO}$ and ${ }^{13} \mathrm{CO}$ for the ${ }^{13} \mathrm{CO}$ lines, from that between ${ }^{13} \mathrm{CO}$ and $\mathrm{C}^{18} \mathrm{O}$ for $\mathrm{C}^{18} \mathrm{O}$, and from that between $\mathrm{C}^{32} \mathrm{~S}$ and $\mathrm{C}^{34} \mathrm{~S}$ for $\mathrm{C}^{34} \mathrm{~S}$. In all cases, such ratios have been computed using the emission integrated under the line and the ${ }^{13} \mathrm{CO}(2-1)$ map has been smoothed to the same angular resolution as the $\mathrm{C}^{34} \mathrm{~S}(3-2)$ map. It is quite clear that the $\mathrm{CO}$ isotopomers trace the roundish, large scale clump and are not sensitive to the small scale, densest parts of it. This can be seen from the fact that the thickest regions in the ${ }^{13} \mathrm{CO}$ and $\mathrm{C}^{18} \mathrm{O}$ transitions are only roughly coincident with the densest cores.

The situation for the $\mathrm{C}^{34} \mathrm{~S}$ line is different. This can be seen more clearly in the top panel of Fig. 16, where the $\mathrm{C}^{32} \mathrm{~S}(3-$ 2) map obtained with the NMA is overlaied to the map of the optical depth in the $\mathrm{C}^{34} \mathrm{~S}(3-2)$ line. In this case, the computation of $\tau$ has been performed using the integral under the red side of the line (from 113 to $116 \mathrm{~km} \mathrm{~s}^{-1}$ ), which is not affected by self-absorption. There is little doubt that the largest column density in the $\mathrm{C}^{34} \mathrm{~S}$ molecule corresponds to the dense cores traced by the $C^{32} S$ emission. By comparing the $C^{32} S$ and $C^{34} S$ maps one gets the impression that the cores are detached from the surrounding gas, which forms a lower density halo around them. We conclude that the $\mathrm{C}^{34} \mathrm{~S}$ region seems to correspond to the transition between the approximate spherical symmetry seen e.g. in the $\mathrm{C}^{32} \mathrm{~S}$ line and the clumpiness due to the formation of the compact cores. This "symmetry break-up" is also outlined by the presence of the bipolar outflows detected in Paper I, which we discuss in the following. 


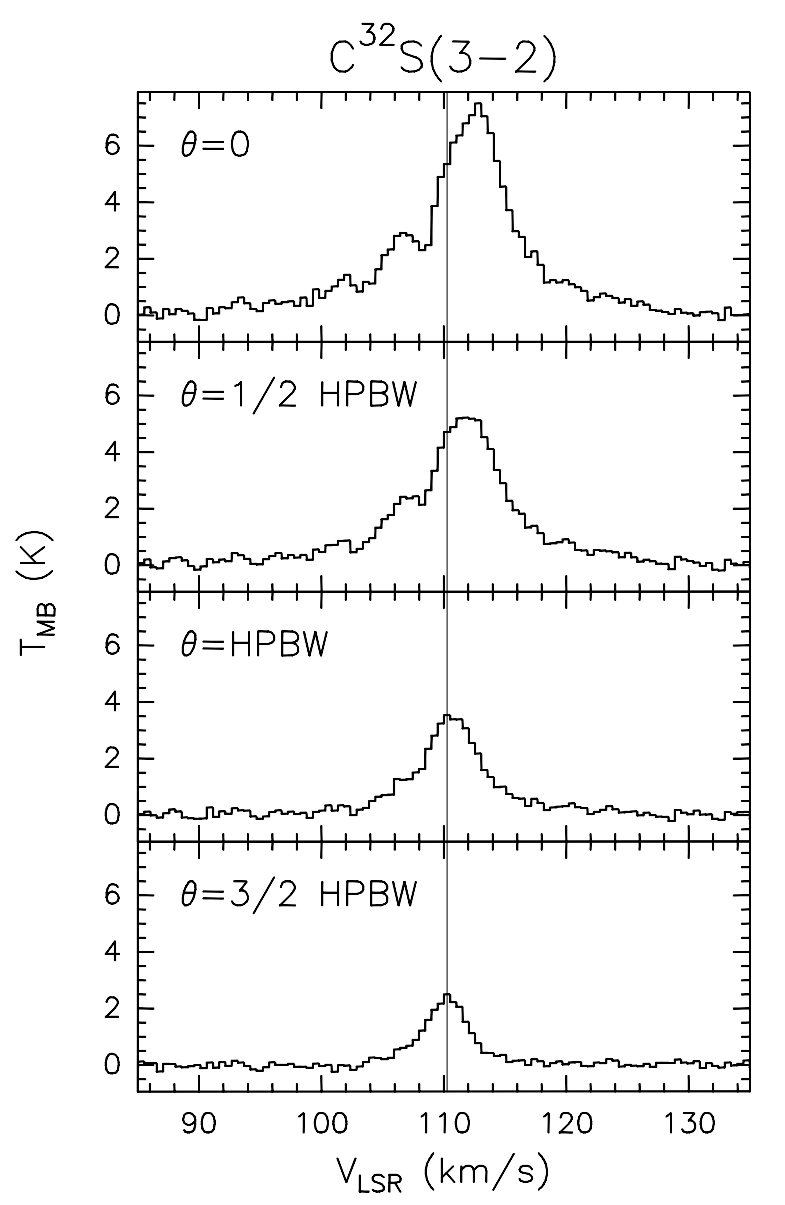

Fig. 13. Mean profiles of the $\mathrm{C}^{34} \mathrm{~S}(3-2)$ line at various offsets, $\theta$, from the clump centre, as indicated in the right corner at the top of each panel. The instrumental $H P B W$ is $16^{\prime \prime}$. The vertical thin line corresponds to the systemic velocity of $110.3 \mathrm{~km} \mathrm{~s}^{-1}$.

\subsection{The outflows}

According to theory (Shu et al. 1987), a clump with density profiles of the type $n_{\mathrm{H}_{2}} \propto R^{-2}$ is bound to undergo inside-out collapse onto a central protostellar core. This process should proceed until all the material has accreted onto the protostar. However, other phenomena can modify this simple picture: among these, outflows are believed to play a fundamental role. In particular, they might reverse the infall and hence halt the collapse thus setting a limit to the mass of the star. In the case of G24.78 two molecular outflows have been found in Paper I in association with cores A and C. The bottom panel of Fig. 16 compares their morphology with a map of the $\mathrm{C}^{34} \mathrm{~S}$ optical depth, $\tau$, over the velocity range $105-116 \mathrm{~km} \mathrm{~s}^{-1}$, namely the whole line profile. Note that this differs from the map in the top panel of the same figure where only the velocity range not affected by self-absorption has been used for the estimate of $\tau$. Clearly, the bottom map peaks towards the blue lobe of the flow from $\mathrm{A}$ : we interpret this as the effect of self-absorption, which causes a decrease of the $\mathrm{C}^{32} \mathrm{~S}$ line intensity at blue-shifted velocities, hence increasing the ratio between $\mathrm{C}^{34} \mathrm{~S}$ and $\mathrm{C}^{32} \mathrm{~S}$ and hence the corresponding estimate of $\tau$. We conclude that only the optical depth map in the top panel of Fig. 16 reflects the column density distribution of the gas: the one in the bottom

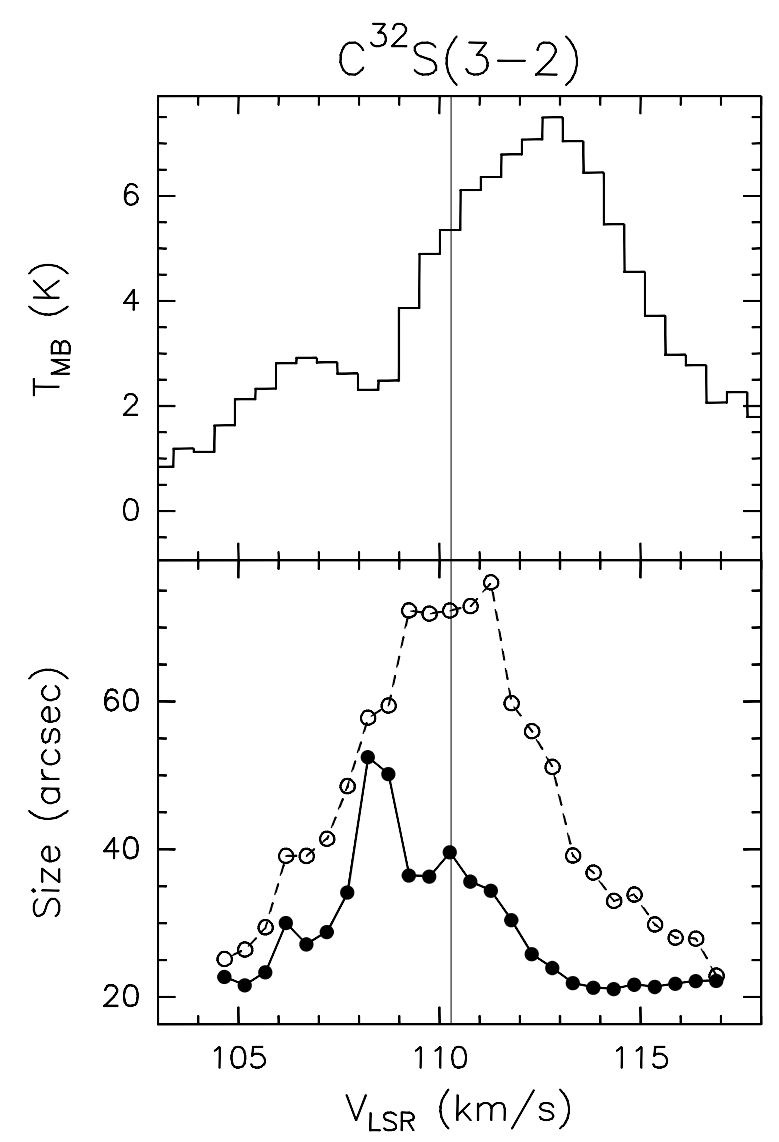

Fig. 14. Top panel: profile of the $\mathrm{C}^{32} \mathrm{~S}(3-2)$ line towards the centre of the clump. Bottom panel: Comparison between the $F W H P$ (full line) and $F W Z I$ (dashed line) of the $\mathrm{C}^{32} \mathrm{~S}(3-2)$ emission maps measured in different velocity channels. The $F W Z I$ is the angular diameter of the contour corresponding to the $3 \sigma$ level in each channel. The vertical thin line marks the systemic velocity of $110.3 \mathrm{~km} \mathrm{~s}^{-1}$.

panel, instead, might be defined as a "pseudo- $\tau$ " map, which indicates where self-absorption is most effective. The fact that the pseudo- $\tau$ peaks towards the blue lobe of the flow from A demonstrates that the expansion seen on a scale of $\sim 1 \mathrm{pc}$ is due to the outflow from A.

The prominence of the outflow from A with respect to that from C is evident also in Fig. 17, which illustrates a comparison between the outflows mapped in different tracers and with different instruments. When observed with high angular resolution (left panels) the ${ }^{12} \mathrm{CO}$ and $\mathrm{C}^{32} \mathrm{~S}$ lines trace both outflows. At low angular resolution, instead, (right panels) the bipolar flow is evident only towards A, which means that the outflow from A is more extended. These characteristics are likely related to a difference in age between the two flows, with that from A being older: such a conclusion is important because it confirms the hypothesis made in Paper I, where we suggested that in G24.78 one is observing a cluster of high-mass YSOs in different evolutionary stages, with $\mathrm{C}$ younger than $\mathrm{A}$.

\section{A model for the G24.78+0.08 star forming region}

Figure 18 is a sketch for the scenario we have in mind for the G24.78 region. One is observing a molecular clump at the 


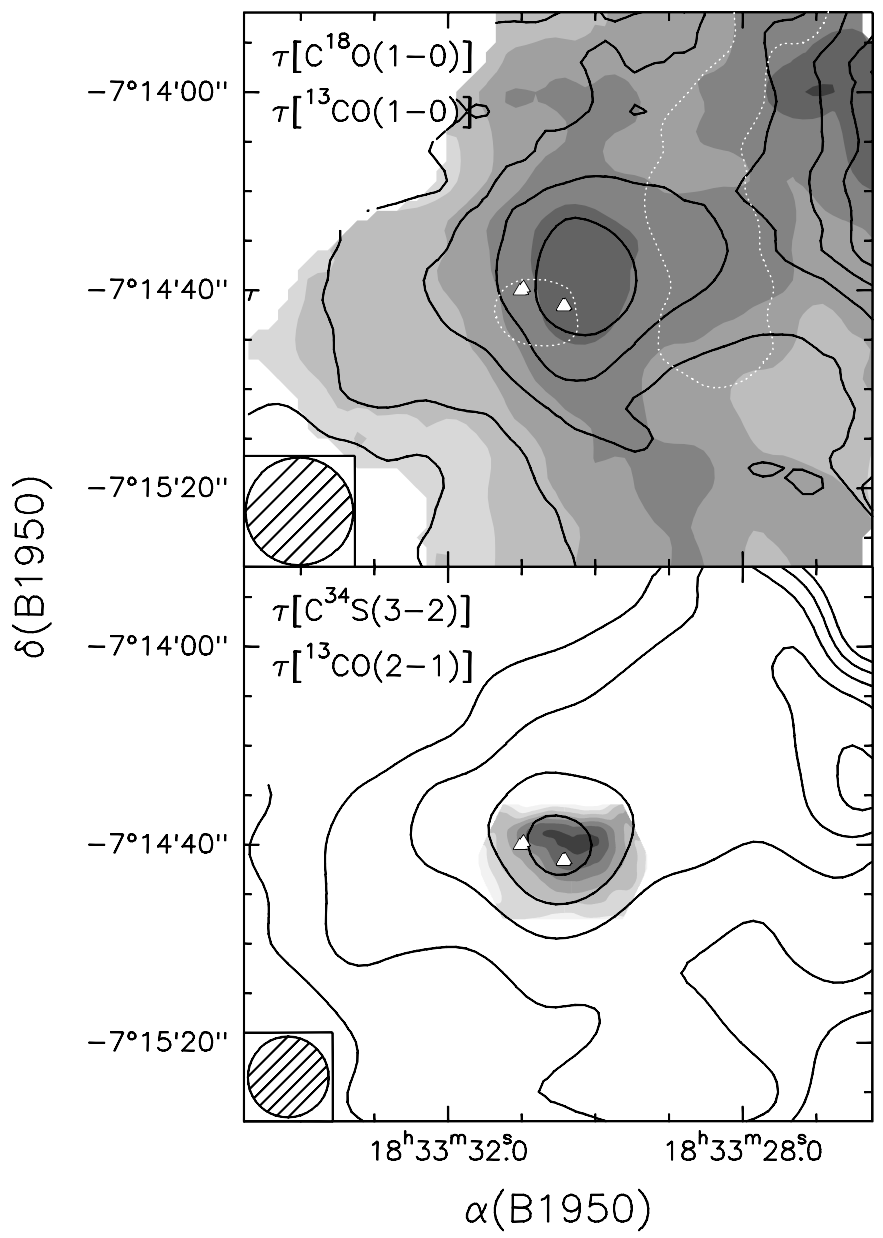

Fig. 15. Top panel: maps of the mean optical depth in the $\mathrm{C}^{18} \mathrm{O}(1-$ $0)$ (grey scale) and ${ }^{13} \mathrm{CO}(1-0)$ (contours) lines over the $V_{\mathrm{LSR}}$ interval $105-116 \mathrm{~km} \mathrm{~s}^{-1}$. Contour levels range from 0.15 to 0.4 in steps of 0.05 for $\mathrm{C}^{18} \mathrm{O}(1-0)$ and from 0.35 to 0.65 in steps of 0.1 for ${ }^{13} \mathrm{CO}(1-$ $0)$. The white dotted contours correspond to the $90 \%$ level of the ${ }^{12} \mathrm{CO}(1-0)$ emission integrated under the line. Bottom panel: same as top panel for the $\mathrm{C}^{34} \mathrm{~S}(3-2)$ (grey scale) and ${ }^{13} \mathrm{CO}(2-1)$ (contours) lines. Contour levels range from 0.4 to 1 in steps of 0.1 for $\mathrm{C}^{34} \mathrm{~S}$ and from 0.3 to 0.7 in steps of 0.1 for ${ }^{13} \mathrm{CO}$. The triangles mark the positions of the $\mathrm{H}_{2} \mathrm{O}$ maser spots. The shaded circles in the bottom left correspond to the instrumental $H P B W$. The ${ }^{13} \mathrm{CO}(2-1)$ map has been smoothed to the same resolution as the $\mathrm{C}^{34} \mathrm{~S}(3-2)$ map.

border of a star forming cloud with filamentary shape. Beside the region of interest for us, early-type stars have already ionised the surrounding gas over a few parsecs. The YSOs observed in Paper I represent the most recent episode of massive star formation in the cloud, as they are still deeply embedded in the parental clump and not associated with extended HII regions, but with cores and UC HII regions.

On a scale of $\sim 1 \mathrm{pc}$ spherical symmetry is a reasonable representation of the gas distribution, whereas inside $\sim 0.2 \mathrm{pc}$ from the centre the symmetry disappears and the material is concentrated in a few dense cores with diameters of $\sim 0.07 \mathrm{pc}$. Such a dishomogeneity is likely to be caused by two processes: collapse and outflow. The former creates the small cores inside which the YSOs have formed. The latter, powered by the YSOs themselves, affects the molecular surroundings by accelerating

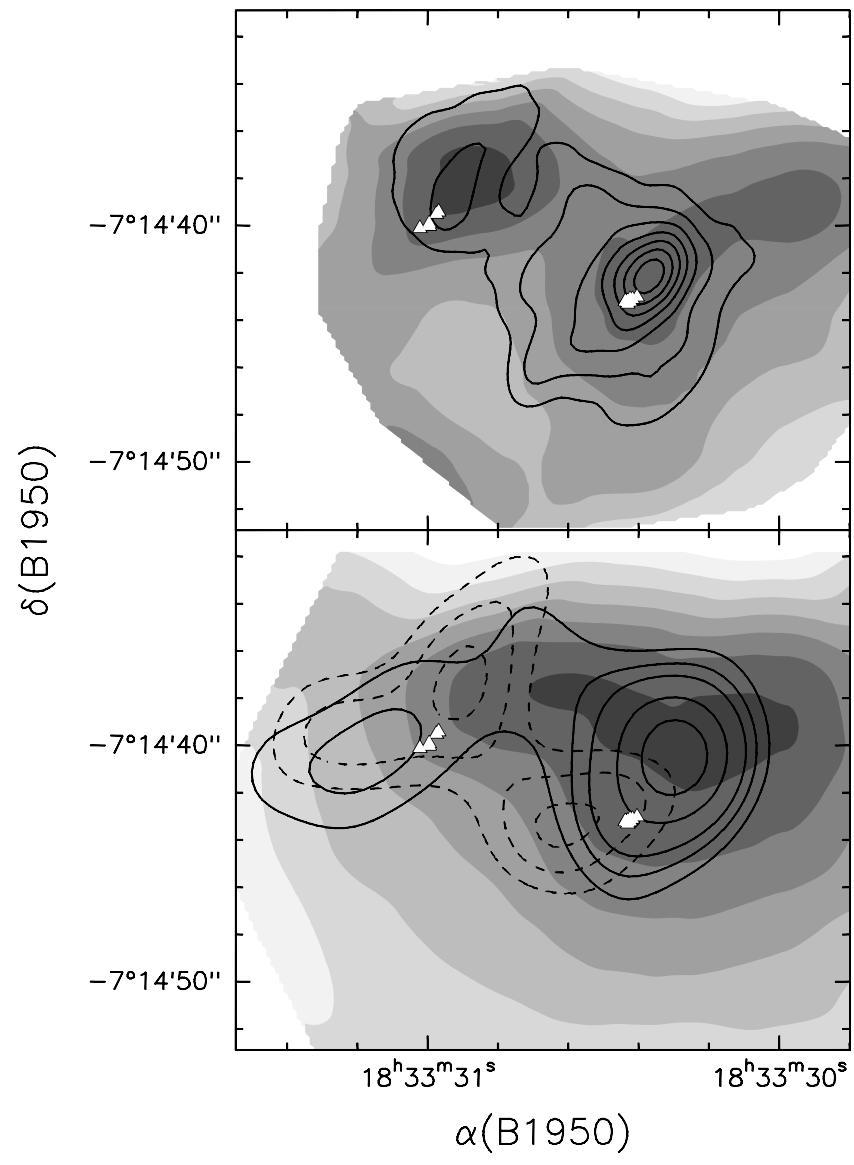

Fig. 16. Top: map of the $C^{34} S(3-2)$ line mean optical depth (grey scale) over the $V_{\mathrm{LSR}}$ range $113-116 \mathrm{~km} \mathrm{~s}^{-1}$, superimposed to the $\mathrm{C}^{32} \mathrm{~S}(3-2)$ emission integrated under the line (contours) observed with the NMA. The triangles mark the positions of the $\mathrm{H}_{2} \mathrm{O}$ maser spots by Forster \& Caswell (1999). Contour levels range from 0.35 to 2.15 in steps of $0.3 \mathrm{Jy} /$ beam. Bottom: map of the $\mathrm{C}^{34} \mathrm{~S}(3-2)$ line mean optical depth (grey scale) over the $V_{\mathrm{LSR}}$ range $113-116 \mathrm{~km} \mathrm{~s}^{-1}$, superimposed to the outflow maps from Paper I. Full and dashed contours represent respectively the blue- and red-shifted ${ }^{12} \mathrm{CO}(1-0)$ line emission integrated under the wings from 90 to $105 \mathrm{~km} \mathrm{~s}^{-1}$ and from 116 to $131 \mathrm{~km} \mathrm{~s}^{-1}$. Contour levels correspond to $1.1,1.4$, and $1.7 \mathrm{Jy} /$ beam (dashed contours) and $0.4,0.6,0.8,1.2$, and $1.6 \mathrm{Jy} / \mathrm{beam}$ (full contours).

part of the clump gas towards the observer: this is seen in blueshifted self-absorption in the optically thickest transitions.

In this scenario, the parsec-scale clump is only weakly affected by the embedded YSOs and may hence be used as a template of the initial conditions prior to the star formation episode. One possibility is that the gas density follows a power law of the type $n_{\mathrm{H}_{2}} \propto R^{-2}$ as expected for a singular isothermal sphere. This configuration is unstable, consistently with the fact that the clump mass is greater than the virial value. As a consequence, inside-out collapse - and star formation - may have occurred inside a radius $R_{\mathrm{c}}$ from the centre, where the theory predicts a density profile $n_{\mathrm{H}_{2}} \propto R^{-1.5}$. As already noted in Sect. 4.1.1, our findings indicate a slope of -1.8 , intermediate between that of the static region and that of the inner collapsing layers. Indeed, in our study of the density we have taken into account line emission from different scales, which might 


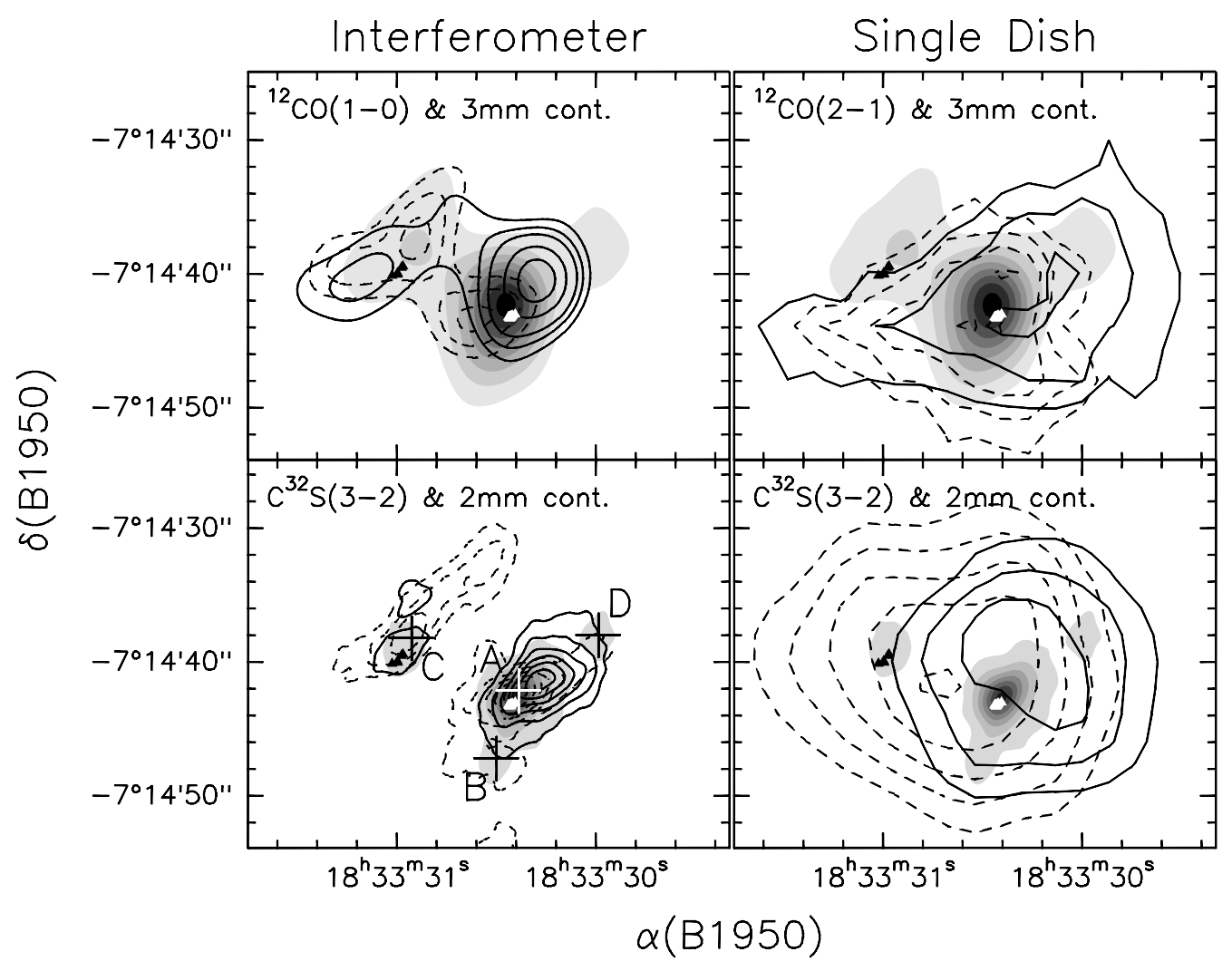

Fig. 17. Left panels: interferometric maps of the emission integrated under the line wings superimposed to the corresponding continuum emission. Information about the line and continuum observed are given in the top left of each panel. Full contours correspond to integrals from 90 to $105 \mathrm{~km} \mathrm{~s}^{-1}$ and dashed contours to integrals from 116 to $131 \mathrm{~km} \mathrm{~s}^{-1}$. Contour levels for ${ }^{12} \mathrm{CO}(1-0)$ are the same as in Fig. 16 (see also Fig. 2 of Paper I); for $\mathrm{C}^{32} \mathrm{~S}(3-2)$ they range from 0.1 to 0.6 in steps of $0.1 \mathrm{Jy} /$ beam. Triangles mark the positions of the $\mathrm{H}_{2} \mathrm{O}$ maser spots. The crosses and corresponding labels indicate the location of the YSOs identified in Paper I. Right panels: same as left panels, for maps obtained with the IRAM 30-m telescope. Contour levels range from 70 to 106 in steps of $12 \mathrm{~K} \mathrm{~km} \mathrm{~s}^{-1}$ for ${ }^{12} \mathrm{CO}(2-1)$ and from 6 to 13.4 in steps of $1.85 \mathrm{~K} \mathrm{~km} \mathrm{~s}^{-1}$ for $\mathrm{C}^{32} \mathrm{~S}(3-2)$.

mix up the contribution from different density laws and hence determine such an "intermediate" slope. If this is the case, one may wonder what is the value of $R_{\mathrm{c}}$, i.e. the radius of the inner collapsing region.

We assume that the material collapsed inside $R_{\mathrm{c}}$ has formed the cores observed in Paper I. The accretion rate $\dot{M} \simeq 3 \times$ $10^{-2} M_{\odot} \mathrm{yr}^{-1}$ can be obtained from Eq. (23) of Shu et al. (1987), adopting a line width $\Delta V_{\frac{1}{2}}=5 \mathrm{~km} \mathrm{~s}^{-1}$ (see Table 2). The age of the cores, i.e. the time since the beginning of the collapse, is hence equal to $t_{\mathrm{c}}=M_{\text {cores }} / \dot{M} \simeq 3 \times 10^{4} \mathrm{yr}$, where the total mass of the cores, $M_{\text {cores }} \simeq 900 M_{\odot}$ is obtained from Table 1 of Paper I. During this period, $R_{\mathrm{c}}$ has moved outward at a speed equal to the measured line width (Shu et al. 1987), so that at present it is equal to $R_{\mathrm{c}}=\Delta V_{\frac{1}{2}} \times t_{\mathrm{c}} \simeq 0.15 \mathrm{pc}$, corresponding to an angular diameter of $8^{\prime \prime 2}$. This is in reasonable agreement with the size of the $\mathrm{C}^{34} \mathrm{~S}$ region where the spherical symmetry break-up is seen. One can compute the infalling mass contained inside $R_{\mathrm{c}}$ assuming that the mean density is that derived from $\mathrm{C}^{34} \mathrm{~S}$ (see Table 2). The result is $\sim 940 M_{\odot}$, indicating that $\sim 50 \%$ of the mass inside $R_{\mathrm{c}}$ has already accreted into the cores, while the remaining $\sim 50 \%$ is still in the collapsing envelope: this is exactly what theoretical models (Shu et al. 1987) predict to happen at any given time during the infall.
In the accretion scenario depicted above, one can speculate that infall does not end onto the stellar surface, but instead creates the dense cores seen in our interferometric maps, which in turn feed the embedded star through, e.g. accretion from a circumstellar disk. Indeed, evidence for slightly flattened, rotating cores has been found in objects similar to G24.78 (see e.g. Cesaroni et al. 1994; Olmi et al. 1996; Cesaroni et al. 1999), mostly through $\mathrm{CH}_{3} \mathrm{CN}$ line emission. Unfortunately, in our case the spectral resolution attained with the NMA observations of the $\mathrm{CH}_{3} \mathrm{CN}(8-7)$ transitions is insufficient to assess whether rotation exists in the cores. However, the data are sufficient to demonstrate that such cores are closer to virialisation than the parsec-scale clump: in fact, the ratio between gas mass and virial mass is $\sim 8.5$ for the $\mathrm{C}^{18} \mathrm{O}$ clump and only $\sim 1.2$ for the $\mathrm{CH}_{3} \mathrm{CN}$ cores (see Table 2). It seems that the innermost part of the clump has reached an equilibrium configuration, as expected for a stationary structure such as, e.g., a rotating disk. Further, higher resolution observations are needed to unambiguously confirm this interpretation.

It must be pointed out that the accretion process must proceed on a free-fall time, with the large mass accretion rate estimated above $\left(\dot{M} \simeq 3 \times 10^{-2} M_{\odot} \mathrm{yr}^{-1}\right)$ : the latter could overwhelm the stellar radiation pressure and hence lead to the 


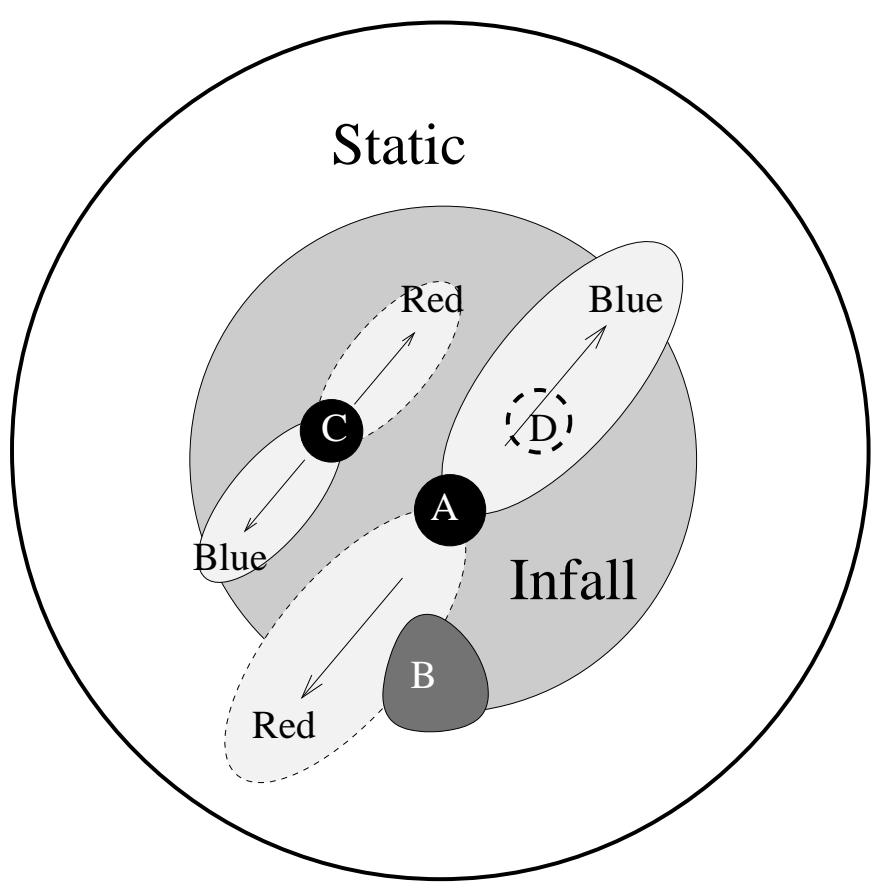

Fig. 18. Sketch of the model for the G24.78 star forming region. The four objects A-D identified in Paper I are embedded in a parsec-scale clump which is undergoing inside-out collapse. Two bipolar molecular outflows with different size are ejected from A and C: these have partly evacuated the infalling region. Object $\mathrm{B}$ is associated with a relatively more evolved UC HII region which according to Codella et al. (1997) is located in the foreground with respect to the clump centre. Protostellar core D is likely to be located behind the blue lobe of the outflow from A.

formation of the high-mass YSOs. Moreover, this could also explain why we do not detect an UC HII region in core $\mathrm{C}$, although according to Paper I this is expected to contain highmass stars: in fact, such an accretion rate is sufficiently large to squelch the ionised region even around an early $\mathrm{O}$ star (see e.g. Walmsley 1995). On the basis of our results one can speculate that the stellar mass will grow until the accretion process is stopped by the outflow powered by the YSOs themselves. As a matter of fact, the outflow age $\left(2 \times 10^{4} \mathrm{yr}\right.$; see Paper I) is very similar to the age of the cores estimated above $\left(3 \times 10^{4} \mathrm{yr}\right)$, consistently with the idea that outflow and accretion are strictly related phenomena. It is also interesting to compare the momentum in the outflow to that in the infall: the former is derived from the parameters of the flows given in Paper I and is equal to $\sim 10^{-3} M_{\odot} \mathrm{yr}^{-1} \times 20 \mathrm{~km} \mathrm{~s}^{-1}=2 \times 10^{-2} M_{\odot} \mathrm{yr}^{-1} \mathrm{~km} \mathrm{~s}^{-1}$; the latter is $\sim 2 \times 10^{-1} M_{\odot} \mathrm{yr}^{-1} \mathrm{~km} \mathrm{~s}^{-1}$, obtained multiplying the accretion rate $\dot{M}$ by the free-fall velocity $\left(\sim 6 \mathrm{~km} \mathrm{~s}^{-1}\right)$ onto $900 M_{\odot}$ from a distance equal to $R_{\mathrm{c}} \simeq 0.2 \mathrm{pc}$. The momentum in the infall is $\sim 10$ times greater than that in the outflows: however, one must take into account that the latter is likely to be underestimated for a variety of reasons. First of all, the interferometer may miss part of the emission from the lobes, as demonstrated by the fact that the single-dish maps in the ${ }^{12} \mathrm{CO}(2-1)$ line give for the outflow from A a mass $\sim 2$ times larger than that estimated from the PdBI maps of the ${ }^{12} \mathrm{CO}(1-0)$ line. Moreover, one cannot exclude that the ${ }^{12} \mathrm{CO}$ transitions might be optically thick even in the line wings. Finally, given the inclination of the flow with respect to the line of sight, the real expansion velocity can be greater than the projected one. One must also take into account the fact that we are observing just the beginning of the star formation process in G24.78: this means that the outflows might become more powerful as the evolution proceeds. Moreover, additional sources might develop outflows, as it is reasonable to foresee in the case of core D: if this contains high-mass protostars, as proposed in Paper I, the latter are bound to contribute to the mass outflow rate in the near future. Therefore, one cannot exclude that outflows might contribute significantly to stop or even reverse the infall, although such a speculation cannot be proved on the basis of the current findings.

In conclusion, the G24.78 star forming region is an excellent laboratory to study the formation process of high-mass stars. Our findings suggest that such a process might occur through inside-out collapse with large accretion rates and subsequent formation of dense molecular cores which represent the cradle of early-type stars. Bipolar outflows are bound to be driven by the YSOs and we speculate that they might stop the accretion as soon as they become sufficiently powerful. This would result in a self-regulating mechanism which allows the formation of massive stars through accretion in a manner alike to that proposed for low-mass stars.

\section{Summary and conclusions}

We have studied the molecular cloud associated with the highmass star forming region $\mathrm{G} 24.78+0.08$. Our analysis has been performed through single-dish and interferometric observations of the continuum and molecular line emission, thus sampling regions from a few parsecs down to $0.1 \mathrm{pc}$. The main findings are as follows:

- The G24.78 region seems to represent the last star formation episode in a filament of molecular gas extending approximately north-south. To the north of such filament an extended HII region is seen, witnessing the presence of older early-type stars.

- A roughly spherical clump is seen in various low-density tracers around the massive YSOs identified in Paper I. Such an approximate spherical symmetry breaks down inside $\sim 0.2 \mathrm{pc}$ from the centre, where dense, compact cores are found.

- The density profile of the clump is well fitted by a power law with slope -1.8 , thus resembling that of a singular isothermal sphere undergoing inside-out collapse.

- We make the hypothesis that the embedded high-mass YSOs might be formed through inside-out collapse. This is supported by the fact that the expected radius of the infalling region is comparable to that of the innermost, heterogeneous region surrounding the cores; also, the mass of such a region is very close to that of the cores, as foreseen for inside-out collapse.

- Single-dish observations in the ${ }^{12} \mathrm{CO}(2-1)$ line demonstrate that the bipolar outflow from core $\mathrm{A}$ found in Paper I is more extended, and hence older, than that from 
core $\mathrm{C}$ : this confirms the evolutionary sequence proposed in Paper I, where C is believed to be younger than A.

- The outflow age is comparable to the time scale required to accumulate the mass of the cores, while the momentum in the flow could be sufficient to stop the accretion rate of $\sim 2 \times 10^{-2} M_{\odot} \mathrm{yr}^{-1}$.

We suggest that G24.78 is an excellent laboratory to study the formation of high-mass stars and the mechanism by which infall is reversed into outflow thus setting the mass of the forming stars. Infall with large accretion rates seems to represent a viable mechanism for high-mass star formation in this region.

Acknowledgements. It is a pleasure to thank the staff of IRAM, NRAO, and NRO for carrying out the observations and help provided during the data reduction. Special thanks are due to Prof. Sachiko Okumura for taking care of our observations with the Nobeyama Millimeter Array.

\section{References}

Blake, G. A., Mundy, L. G., Carlstrom, J. E., et al. 1996, ApJ, 472, L49

Cesaroni, R., Walmsley, C. M., Kömpe, C., \& Churchwell, E. 1991, A\&A, 252, 278
Cesaroni, R., Olmi, L., Walmsley, C. M., Churchwell, E., \& Hofner, P. 1994, ApJ, 435, L137

Cesaroni, R., Felli, M., Jenness, T., et al. 1999, A\&A, 345, 949

Codella, C., Testi, L., \& Cesaroni, R. 1997, A\&A, 325, 282

Condon, J. J., Cotton, W. D., Greisen, E. W., et al. 1998, AJ, 115, 1693

Felli, M., Palagi, F., \& Tofani, G. 1992, A\&A, 255, 293

Fontani, F., Cesaroni, R., Caselli, P., \& Olmi, L. 2002, A\&A, 389, 603

Forster, J. R., \& Caswell, J. L. 1989, A\&A ,213, 339

Forster, J. R., \& Caswell, J. L. 1999, A\&AS, 137, 43

Furuya, R. S., Cesaroni, R., Codella, C., et al. 2002, A\&A, 390, L1 (Paper I)

Irvine, W. M., Goldsmith, P. F., \& Hjalmarson, H. 1987, Interstellar Processes, ed. D. J. Hollenbach, \& H. A. Thronson, 561

Li, Z. 1999, ApJ, 526, 806

Moscadelli, L., Cesaroni, R., \& Rioja, M. J. 2000, A\&A, 360, 663

Okumura, S. K., Momose, M., Kawaguchi, N., et al. 2000, PASJ, 52, 393

Olmi, L., Cesaroni, R., \& Walmsley, C. M. 1993, A\&A, 276, 489

Olmi, L., Cesaroni, R., Neri, R., \& Walmsley, C. M. 1996, A\&A, 315, 565

Palla, F., \& Stahler, S. W. 1993, ApJ, 418, 414

Shu, F. H., Adams, F. C., \& Lizano, S. 1987, ARA\&A, 25, 23

Stahler, S., Palla, F., \& Ho, P. T. P. 2000, in Protostars and Planets IV, ed. V. Mannings, A. Boss, \& S. Russel (Tucson: Univ. of Arizona Press), 327

Walmsley, C. M. 1995, Rev. Mex. Astron. Astrofis., Ser. Conf., 1, 137

Wilner, D. J., \& Welch, W. J. 1994, ApJ, 427, 898

Wilson, T. L., \& Rood, R. 1994, ARA\&A, 32, 191 